\title{
Network Amplification of Local Fluctuations Causes High Spike Rate Variability, Fractal Firing Patterns and Oscillatory Local Field Potentials
}

\author{
Marius Usher \\ Martin Stemmler \\ Christof Koch \\ Computation and Neural Systems, 139-74, California Institute of Technology, \\ Pasadena, CA 91125 USA
}

\section{Zeev Olami}

Department of Chemical Physics, Weizmann Institute of Science, Rehovot 76100, Israel

We investigate a model for neural activity in a two-dimensional sheet of leaky integrate-and-fire neurons with feedback connectivity consisting of local excitation and surround inhibition. Each neuron receives stochastic input from an external source, independent in space and time. As recently suggested by Softky and Koch (1992, 1993), independent stochastic input alone cannot explain the high interspike interval variability exhibited by cortical neurons in behaving monkeys. We show that high variability can be obtained due to the amplification of correlated fluctuations in a recurrent network. Furthermore, the crosscorrelation functions have a dual structure, with a sharp peak on top of a much broader hill. This is due to the inhibitory and excitatory feedback connections, which cause "hotspots" of neural activity to form within the network. These localized patterns of excitation appear as clusters or stripes that coalesce, disintegrate, or fluctuate in size while simultaneously moving in a random walk constrained by the interaction with other clusters. The synaptic current impinging upon a single neuron shows large fluctuations at many time scales, leading to a large coefficient of variation $\left(C_{V}\right)$ for the interspike interval statistics. The power spectrum associated with single units shows a $1 / f$ decay for small frequencies and is flat at higher frequencies, while the power spectrum of the spiking activity averaged over many cells-equivalent to the local field potential-shows no $1 / f$ decay but a prominent peak around $40 \mathrm{~Hz}$, in agreement with data recorded from cat and monkey cortex (Gray et al. 1990; Eckhorn et al. 1993). Firing rates exhibit self-similarity between 20 and $800 \mathrm{msec}$, resulting in $1 / f$-like noise, consistent with the fractal nature of neural spike trains (Teich 1992). 


\section{Introduction}

A puzzling conflict between standard biophysical theories and the characteristics of spike trains recorded from cortical cells responding at high rates to visual input has recently been pointed out (Softky and Koch 1993). Experimental evidence shows that the amplitude of an individual excitatory postsynaptic potential (EPSP) is on the order of $0.1 \mathrm{mV}$, about two orders of magnitude smaller than the threshold depolarization from rest necessary for a pyramidal cell to spike (Komatsu et al. 1988; Mason et al. 1991) [for a review see also (Fetz et al. 1991)]. Based on this, Softky and Koch showed that the neural firing pattern will be highly regular if the neuronal membrane acts as a leaky integrator summing over a train of stochastic, uncorrelated EPSPs. In an integrator model, the time to spike is determined by the total time in which a critical number of EPSPs accumulate. Since the interspike interval is the sum of random variables representing the intervals between EPSP inputs, the central limit theorem predicts that the output spikes will be highly regular. In other words, the shape of the interspike interval histogram will become highly peaked as measured by the coefficient of variation, $C_{V}$, defined as the standard deviation over the mean of the interspike interval (ISI) distribution. Softky and Koch also showed that this central limit result holds for a detailed biophysical compartmental model (including seven voltage-dependent somatic currents) of a cortical pyramidal cell in the presence of independent synaptic input. However, recordings from cells in V1 and MT cortex in the behaving monkey show that the discharge at high rates (up to $200 \mathrm{~Hz}$ ) is highly variable in the length of interspike intervals, with a coefficient of variation of around one.

Softky and Koch (1993) suggested two possible solutions to this dilemma: the first one requires fast and powerful active $\mathrm{Na}^{+}$conductances sensitive to inputs at the millisecond time scale in the dendrites (as discussed in depth by Softky 1993). In this framework, neurons act as coincidence detectors, firing only if many synaptic inputs arrive simultaneously (Abeles 1982, 1991). A second approach, which we will develop here, is to solve the discrepancy by challenging the assumption of uncorrelated inputs while preserving the standard biophysical model of a temporally integrating membrane summing over small EPSPs. Here, interspike interval variability is a direct consequence of the global network dynamics, which create and amplify correlated fluctuations in an irregular fashion. This approach is motivated by the fact that extensive axon collaterals of pyramidal cells in cortex permit massively recurrent connections between neurons, which of necessity leads to strong correlations in the spike output of cells in the same proximity; however, recurrent connections alone do not lead to an increase in variability. In fact, depending on the pattern of connections, the opposite may occur. For example, in a recurrent network cells may entrain themselves into a steady state fixed point or to a limit cycle (oscillation) (Amit and Tsodyks 1991a,b; Koch and Schuster 
1992; van Vreeswijk and Abbott 1993), where the interspike variability would be even lower than for uncorrelated input.

We find that the generation of high variability depends critically on the spatial extent of inhibition relative to excitation. One connectivity pattern that robustly results in high variability is local excitation surrounded by inhibition, isotropically distributed across the entire network. Theoretical investigations of such connectivity using continuous firing rate models have shown that the translational and rotational symmetry of an isotropic network can break spontaneously, leading to localized patterns of excitations (Willshaw and von der Malsburg 1976; Amari 1977; Wilson and Cowan 1973; Ermentrout and Cowan 1980; Chernjavsky and Moody 1990) [see also Cowan (1982) for a bifurcation analysis predicting various geometric patterns, such as hexagonal or square lattices]. Generalizing this approach to a spiking model with noisy input, we find that the patterns of excitation exhibit metastability with large temporal fluctuations.

In particular, we find that in the presence of homogeneous and independent spatiotemporal input to all cells firing patterns display fluctuations on many temporal scales, leading to $1 / f$ components in the power spectra, and high variability in the number of events. Furthermore, correlation functions between neighboring units display a sharp peak around zero, riding on a much broader hill. Such fluctuations of the firing behavior across many different time scales have recently been reported in neurons from the auditory pathway in mammals and the mesencephalic reticular formation by (Teich 1989, 1992; Grueneis et al. 1990), who showed that it implies temporal clustering of events and a fractal firing pattern.

In the following, we first review different aspects of the variability problem. In Section 3, our model is presented, followed by the results in Section 4 . Finally, we discuss the relationship between our results and typical electrophysiological experiments in monkey cortex as well as the implications of our model in the last section.

\section{The Variability Problem}

2.1 Interspike Variability. The interspike interval histogram and the coefficient of variation can be easily obtained for a pure integrator that receives a stream of Poisson inputs. Consider first the case of pure excitation in which an integrate-and-fire unit receives Poisson distributed EPSPs at rate $\lambda$. Assuming that the membrane potential is reset to zero after each emitted spike and that $N_{\theta}$ synaptic inputs are required in order for the unit to reach threshold, the output interspike interval distribution $P_{N_{\theta}}(T)$ is equal to the probability that $N_{\theta}$ events will arrive during an interval $T$. If the arrival times of the incoming EPSPs are exponentially distributed, $P(T)=\lambda \exp (-\lambda T)$, then the probability distribution for the 


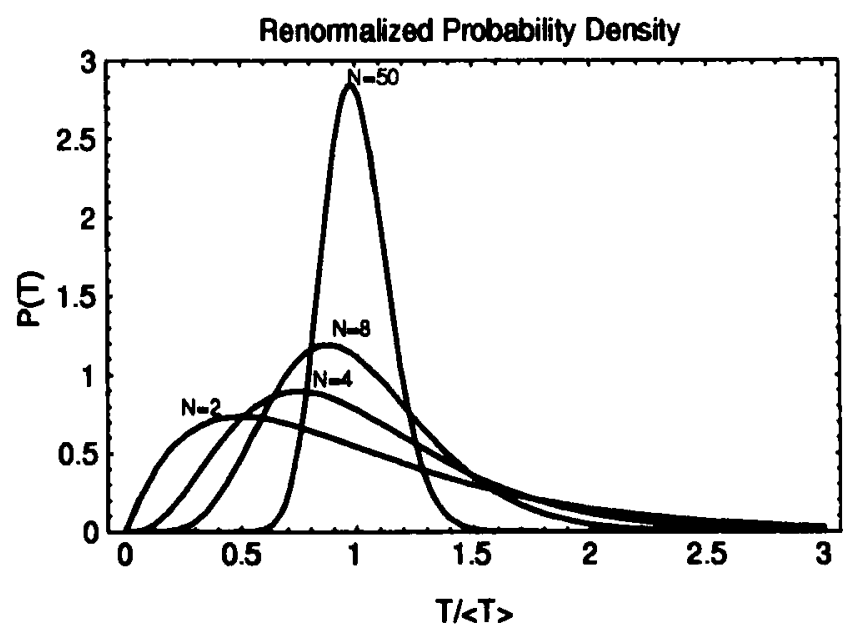

Figure 1: Probability density functions of $P_{N_{\theta}}$ for an integrate-and-fire unit requiring $N_{\theta}$ Poisson-distributed synaptic inputs to fire. The interspike interval (ISI) distribution becomes relatively narrower as the number of inputs required to reach threshold increases, a consequence of the Law of Large Numbers. The $x$-axis is in units of the mean time to spike $\langle T\rangle$. Functions are rescaled so that integrals under curves are all equal to unity.

sum of $N_{\theta}$ independent identically distributed random variables, $P_{N_{\theta}}(T)$ is

$$
P_{N_{\theta}}(T)=\lambda \frac{(\lambda T)^{N_{\theta}-1}}{\left(N_{\theta}-1\right) !} \exp (-\lambda T)
$$

If we denote the mean time between spikes as

$$
\langle T\rangle=\frac{N_{\theta}}{\lambda}
$$

(where \langle\rangle indicates the temporal average), we can introduce the normalized probability distribution $P_{N_{\theta}}(T /\langle T\rangle)$. Although $P_{N_{\theta}}(T)$ becomes broader with increasing $N_{\theta}$, the normalized distribution $P_{N_{\theta}}(T /\langle T\rangle)$ becomes narrower. In Figure 1, we display $P_{N_{\theta}}(T /\langle T\rangle)$ for several values of $N_{\theta}$, assuming a constant rate $\lambda$ of inputs.

From equation 2.1, we obtain the variance and the standard deviation divided by the mean (the coefficient of variation $C_{V}$ ):

$$
\begin{aligned}
\operatorname{Var}(T) & =\left\langle(T-\langle T\rangle)^{2}\right\rangle=\frac{N_{\theta}}{\lambda^{2}} \\
C_{V} & =\frac{\operatorname{Var}(T)^{1 / 2}}{\langle T\rangle}=\frac{1}{\sqrt{N_{\theta}}}
\end{aligned}
$$


We observe that the coefficient of variation decreases with the square root of $N_{\theta}$. Thus for $N_{\theta}=100$, corresponding to a reasonable estimate of the total number of EPSPs required to trigger a cortical pyramidal cell (Komatsu et al. 1988; Mason et al. 1991), one obtains $C_{V}=0.1$, much lower than the experimental values for cortical cells firing at high rates, for which $C_{V} \approx 1$. This result is simply a consequence of the Law of Large Numbers: the sum of a sufficiently large number of independent random variables has a gaussian distribution.

In principle, the variability can increase due to inhibition, which may cancel part of the mean rate of the input signal while keeping or increasing the fluctuation. However, a significant contribution of inhibition to variability can be ruled out for biophysical and mathematical reasons.

First, compartmental simulations (Softky and Koch 1993) show that the degree of inhibition required for this is much larger than observed in in vivo intracellular recordings in cells in cat striate cortex (Douglas et al. 1988; Berman et al. 1991). In the following, we briefly outline a mathematical argument.

Consider a cell receiving a superposition of an excitatory Poisson stream (of rate $\lambda_{e}$ ) and an inhibitory stream (of rate $\lambda_{i}$ ) of synaptic inputs, of equal but opposite magnitude. Under these conditions, the interspike interval, interval's mean and variance can be written as (Tuckwell 1988):

$$
\begin{aligned}
\langle T\rangle & =\frac{N_{\theta}}{\lambda_{e}-\lambda_{i}} \\
\operatorname{Var}(T) & =\frac{N_{\theta}\left(\lambda_{e}+\lambda_{i}\right)}{\left(\lambda_{e}-\lambda_{i}\right)^{3}}
\end{aligned}
$$

The coefficient of variation is:

$$
C_{V}=\frac{1}{\sqrt{N_{\theta}}} \sqrt{\frac{\left(\lambda_{e}+\lambda_{i}\right)}{\left(\lambda_{e}-\lambda_{i}\right)}}
$$

Equation 2.4 shows that in order to obtain a $C_{V}$ of order of one, the relation between excitation and inhibition rates should satisfy

$$
\frac{\left(\lambda_{i}+\lambda_{i}\right)}{\left(\lambda_{i}-\lambda_{i}\right)} \approx N_{\theta}
$$

or in terms of the rate of inhibition,

$$
\lambda_{i} \approx \frac{N_{\theta}-1}{N_{\theta}+1} \lambda_{e}
$$

For $N_{\theta}=100$, the inhibition rate should equal $\lambda_{i}=0.98 \lambda_{e}$. In other words, both excitation and inhibition need to be roughly 50 times larger than the net resulting current proportional to $\lambda_{e}-\lambda_{i}$. No experimental evidence exists for such extremely high background currents. This scheme requires an unusually high degree of balancing, since a mere $2 \%$ higher inhibitory rate would totally cancel the excitatory input. Finally, 
the output rate of such a neuron would be 50 times lower than the input rate, since the mean output rate is given by $\left(\lambda_{e}-\lambda_{i}\right) / N_{\theta}=0.02 \lambda_{e} / N_{\theta}$.

When membrane leakage is taken into account, the interspike interval histogram associated with the leaky integrate-and-fire unit cannot be derived analytically. However, as shown by Softky and Koch (1993) in simulations of both integrate-and-fire and a detailed compartmental model, leakage leads to an increase in variability only for very low firing rates (relative to the reciprocal of the membrane time constant). At high output rates (relative to the inverse of the membrane time-constant), no significant decrement occurs between spikes, and the $C_{V}$ is not affected. Because cortical recordings show large coefficients of variation even at high firing rates (Softky and Koch 1993), the problem of variability requires a different solution.

2.2 Variability in the Number of Events. A different measure of the "unpredictability" of a cell's discharge is the variability in the number of action potentials the cell fires in response to specific input.

If action potentials are distributed according to a Poisson point process, the simplest of all stochastic processes, the mean number of spikes, should be identical to the variance: $\operatorname{Var}(N)=N$. Yet, experimentally, neurons frequently show larger fluctuations, indicating clustering of the spike trains. Two different experimental paradigms have been used to evaluate this.

In the first paradigm, the mean discharge rate associated with one cell respunding numerous times to one particular stimulus for a fixed interval is computed along with the variance about the mean, resulting in one $\left[N_{i}, \operatorname{Var}\left(N_{i}\right)\right]$ pair (Snowden et al. 1992; Vogels et al. 1989; Tolhurst et al. 1983). This experiment is repeated for different stimuli and different cells (always for the same duration). These pairs are then plotted in log-log coordinates. For a Poisson process, the slope of the line passing through these points should be 1 . Yet, different experiments in both V1 and in MT of the anesthetized or the behaving monkey using bars, gratings or random-dot stimuli have consistently found a scaling law of the type $\operatorname{Var}(N) \approx N^{5 / 4}$. In other words, the variance in the number of spikes is greater than expected for a pure Poisson process.

A clue to the origin of this high variability comes from a related experiment, in which long spike trains from neurons responding either spontaneously or to stationary sensory stimuli are partitioned into nonoverlapping time windows $T$, for which then the mean and variance in the number of events are calculated as a function of $T$. When the spike discharge in response to a constant input in different noncortical neurons is evaluated in this manner, the neurons show high variability, leading to a power law of the form:

$$
\operatorname{Var}[N(T)] \propto[N(T)]^{\nu}
$$


Again, for a pure Poisson process, $\nu=1$. Sometimes (Teich 1992) an equivalent exponent, called the Fano number, equal to $\nu-1$, is used instead. For regular spike trains, whose mean interspike interval does not diverge, the exponent $\nu$ has an upper bound of 2 (exponents larger than two lead to large and nonconvergent fluctuations in the firing rate). On the other hand, exponents $\nu<1$ imply high regularity in the firing pattern (e.g., a purely periodic spike train has zero variance and hence $\nu=0$ ). Typical values for $\nu$ range from 1.2 to 2.0 for spike trains from auditory nerve fiber (Teich 1989, 1992) and the cochlear nucleus (Shofner and Dye 1989), ${ }^{1}$ while the firing of vestibular neurons is much more regular, with $\nu=1.03$ (Teich 1992).

What are the implications of these power laws? Furthermore, what is the relationship between assessing the mean and variability in a neuron's response by keeping the time window $T$ constant while varying the stimulus intensity (or contrast) and, in a different experiment, varying $T$ while keeping the stimulus constant?

The implication of $\nu>1$ in equation 2.6 can be understood by examining the fluctuations in the firing rate $f=N(T) / T$, which can be described in terms of $\Delta N(T)=\sqrt{\operatorname{Var}[N(T)]}$ :

$$
\begin{aligned}
\Delta f & =\frac{\Delta N(T)}{T} \\
& \propto N^{\nu / 2-1}
\end{aligned}
$$

For a Poisson process, where $\nu=1$, the rate fluctuations decay with increasing time windows as $T^{-1 / 2}$. That is, recording four times longer doubles the signal-to-noise ratio. Values of $\nu$ bigger than 1 imply persistent firing rate fluctuations and clustering of the spiking process: fluctuations in the instantaneous firing rate will be partially preserved when the rate is averaged over increasingly longer intervals. In the extreme case of $\nu=2$, the ratio of signal to noise remains constant no matter how long one averages.

The random walk described by the deviation from the mean number of spikes in an interval $T$, that is $N(T)-\langle N(T)\rangle$, leads to a different way to understand the significance of the $\nu$ exponent. The standard deviation of this walk is proportional to $T^{\nu / 2}$. Thus, multiplying the time interval by a factor of 2 scales the walk deviation by a factor of $2^{\nu / 2}$. Such processes are called self-affine fractals, and $\nu / 2$ is called the roughness (or Hurst) exponent (Feder 1988). For the case of $\nu=2$, the walk is itself-similar and scales by the same factor as the time interval, showing self-similarity.

Two important statistical measures of spike trains related to the existence of self-similarity and persistent fluctuations, as reflected in the power law discussed above, are the interspike interval distribution $P(t)$

\footnotetext{
II a preliminary study, we have found similar power-law exponents for long spike trains recorded from the parietal cortex of monkeys performing delayed matching to sample tasks in the laboratory of J. Fuster (Zhou and Fuster 1992).
} 
and the autocorrelation function $A(t)$. These two measures relate complementary statistical properties of spike trains: while $P(t)$ measures statistics of intervals between consecutive spikes and is order independent, $A(t)$ measures the fraction of spikes separated by time $t$ and is related to statistics of intervals of all orders.

While a power law decay (with exponent between 0 and 1 ) in the autocorrelation function implies that the spike train fluctuations in $N(t)$ are persistent and self-affine (characterized by $\nu$ exponents between 1 and 2), we shall show that a power law decay (with an exponent between 1 and 2) in the interspike interval distribution indicates that the point process is a true fractal with a noninteger dimension $D$ between 0 and 1 .

Consider first the interval distribution $P(t)$. Following Mandelbrot (1983), the fractal dimension of an infinite recursive point process (dust) is defined via the number of covering intervals of length $\delta, N(\delta)$, needed to cover all the events in a finite interval of length $T$ :

$$
N(\delta) \times \delta^{-D}
$$

Since actual spike trains have finite length, equation 2.7 can hold only over a limited temporal range. Clearly, when $\delta$ is much smaller than the mean interspike interval, the number of covering intervals saturates at the total number of spikes in the train, imposing a lower cutoff on the range over which the process exhibits power law behavior.

We show in Appendix $A$ that a point process whose interspike interval distribution decays as a power-law with exponent $-\gamma$, satisfying $1 \leq \gamma \leq 2$, has a fractal dimension $D=\gamma-1$. Thus processes whose interval distribution decays faster than $t^{-2}$, such as the Poisson process whose interval distribution decays exponentially, have a dimension of one, implying that at time-scales longer than the mean interval there are very few empty covering intervals. On the other hand, for a power law distribution with $1<\gamma<2$, the process is much more clustered, resulting in a large number of empty covering intervals. The dimension $D$ for such clustered processes is a noninteger number that lies between zero and one.

The second statistical measure $A(t)$ can be related to the variance in the number of events $N(T)$ and the $\nu$ exponent (Cox and Lewis 1966; Teich 1989). For any stationary point process of mean rate $\lambda$, the variance in the number of events in time $T$ (see Appendix $B$ ) is

$$
\operatorname{Var}(N)=N+2 \int_{0}^{T}(T-t)[A(t)-A(\infty)] d t
$$

In the absence of correlations $[A(t)=A(\infty)]$ for all values of $t$, this equation implies that $\operatorname{Var}(N)=N$, as for a Poisson process. If the autocorrelation function decays to chance levels in finite time $t_{c}$, the variance will be proportional to $N$ for $t>t_{c}$. The power law behavior for $N(T)$ will be observed, therefore, only if there are long-range temporal correlations in the data. 
Table 1: Scaling Relations.

\begin{tabular}{cccc}
$\operatorname{Var}(N)$ & Autocorrelation & Power spectrum & ISI distribution \\
\hline $\operatorname{Var}(N) \sim N^{\nu}$ & $A(t) \sim t^{\nu-2}$ & $S(f) \sim f^{-\nu+1}$ & $P(t) \sim t^{-1}$ \\
\hline
\end{tabular}

Since the number of spikes $N$ depends linearly on $T$, equation 2.8 will lead to $\operatorname{Var}[N(T)] \propto T^{\prime \prime}$ (for large values of $N$ ) only if the autocorrelation function satisfies

$$
A(t)-A(\infty) \propto t^{\prime \prime-2}
$$

for large values of $t^{2}$

By the Wiener-Khinchin theorem, the power spectrum of a process is the Fourier transform of the autocorrelation. Assuming that $A(t) \gg$ $A(\infty)$, equation 2.9 leads to

$$
S(f) \propto \lambda f^{-1+1}
$$

for small frequencies. For $1 \leq \nu \leq 2$, the exponent of the power spectrum at low frequencies will be between minus one and zero. Such exponents in the power spectrum are generally called $1 / f$ noise.

For the special case of a renewal process, in which consecutive intervals are independent of each other, a power law with exponent $-\gamma$ in $P(t)$ leads to a power law with exponent $\gamma-2$ in the autocorrelation function $A(t)$ (Lowen and Teich 1993). For such processes, the exponent $D$ in equation 2.7 is related to the Hurst or roughness exponent $\nu / 2$ by

$$
\nu=D+1=\gamma
$$

In the strictest sense, these power law relationships hold only in the limit for which $P(t)$ behaves as a power law for all $t$. If the power law behavior extends only over a finite range, the prediction of exponents becomes approximate. For renewal processes characterized by a power law between two temporal cut-offs, full analytic solutions of $A(t)$ given $P(t)$ are difficult to obtain [but see Lowen and Teich (1993) for the special case of $D=0.5$ ]. A restricted range of power law behavior is in general accompanied by a significant baseline autocorrelation $A(\infty)$, so that if $A(t)$ decreases as a power law, $A(t)-A(\infty)$ will, of course, decrease more slowly than $A(t)$. By the same token, $\operatorname{Var}(N)$ will increase more slowly with $N$ than predicted.

We summarize the main scaling relationships in Table 1.

${ }^{2}$ If $A(t) \propto e^{-t / r^{\prime}}$, then $\operatorname{Var}(N) \times N$ in the asymptotic limit of long $T$. Only $A(t) \times t^{-3}$ leads to a power law for $\operatorname{Var}(N)$ valid at large $N$. 
These power law relationships will also hold-under certain conditions-for the first type of experiment (with fixed recording time $T$, but variable stimulus intensity leading to corresponding mean rates $\lambda$ ). In this case, the result

$$
\operatorname{Var}(N)=N^{\nu^{\prime}}
$$

with $1 \leq \nu^{\prime} \leq 2$ can be explained in terms of equation 2.8 if

$$
A(t, \lambda) \propto \lambda^{\nu^{\prime}} A(t)
$$

in the simultaneous presence of long-range temporal correlations. In other words, if the correlation function scales in this manner with the mean firing frequency and the temporal correlations are long-lasting, equation 2.8 implies $\operatorname{Var}(N) \propto N^{\nu^{\prime}}$ for large values of $N$ (for fixed time intervals $T$ ).

Note that there is no a priori reason for the exponent $\nu^{\prime}$ obtained in the first experimental paradigm to be equal to $\nu$ measured in the second paradigm. If, however, the normalized correlation between spikes separated by time $t$ is only a function of the expected number of intervening spikes in time $t, \lambda t$, the two exponents will, in fact, be equal. This condition translates into

$$
A(t, \lambda) \propto \lambda^{\mu} t^{\nu-2}
$$

implying that the autocorrelation function scales with the mean firing rate. In particular, this condition holds for a perfect integrate-and-fire unit, since rescaling the input rate will only change the output rate of such a unit, and not the relative temporal ordering of output spikes. Thus scaling the input rate is equivalent to a rescaling of time (playing the recorded tape at a different speed), and, therefore, the natural quantity for measuring the autocorrelation decay is not the absolute time itself, but rather the intervening number of spikes, $t \lambda$.

The origin of long-range temporal correlations in cortical spike trains remains a mystery. The emergence of such long range correlations is considered anomalous in most physical systems, where they generally occur only at a "critical point" during a phase transition. Dynamic systems that robustly produce such behavior by self-organization have recently been introduced into the literature under the label of "self-organized criticality" (Bak et al. 1987; Olami et al. 1992). In the following, we propose a neural model based on a self-organizing dynamic metastable system, which provides a solution to both aspects of the variability problem.

\section{The Model}

We searched for the simplest network that explains high output variability while still adhering to the fundamental constraints of cortical anatomy 
and electrophysiology. Consequently, we used as our standard setup a model based on leaky integrate-and-fire neurons with semilocal connectivity. Modifications of this standard setup have also been studied and will be described below. We first describe the neural dynamics and subsequently the connectivity patterns used in the simulations. Although not necessary, it is helpful for the remainder of the paper to think of the simulated network as a sheet of cortical neurons in primary visual cortex receiving unstructured visual input from the lateral geniculate nucleus.

3.1 Neural Dynamics. The model consists of a two-dimensional lattice of units, connected within the layer by local excitatory and inhibitory synapses. Each unit integrates the inputs with a time constant $\tau$ characterizing the passive neuronal membrane; once the potential reaches the threshold voltage $V_{\theta}$, the unit emits a spike that is transmitted to synaptically connected neighboring units, and the potential is reset to zero.

The dynamics of the integrate-and-fire model are given by

$$
\begin{aligned}
& \frac{d V_{i}(t)}{d t}=\frac{-V_{i}(t)}{\tau}+I_{i}\left(t-t_{\text {delay }}\right) \\
& I_{i}(t)=\sum_{j} J_{i j}^{E} \theta\left[V_{j}(t)-V_{\theta}\right]+I_{i}^{\text {ext }}+\sum_{j} I_{i j}^{I} \theta\left[V_{j}(t)-V_{\theta}\right]
\end{aligned}
$$

with

$$
\begin{array}{ll}
J^{E}=\alpha / N_{\text {con }} & \text { excitatory synaptic coupling strength } \\
J^{\prime}=\beta J^{E} & \text { inhibitory coupling strength }(\beta<1)
\end{array}
$$

where the indices $i$ and $j$ denote the units, $t_{\text {delay }}$ is a transmission delay, $\theta$ is the Heaviside step function $[\theta(x)=1$ for $x>0$ and $\theta(x)=0$ otherwise], and $I^{\text {ext }}$ is the external current impinging upon the cell.

Some simulations used a biophysically correct model of synaptic input as conductance changes in series with ionic reversal batteries. The effective driving potential is thus given by the difference between the voltage and the reversal potential, that is, $E_{\mathrm{exc}}-V_{i}(t)$. $E_{\mathrm{exc}}$ was set at five times the threshold voltage, corresponding to fast voltage-independent AMPA excitatory input, while $E_{\text {inh }}$ was set to be shunting or silent, that is, $E_{\text {inh }}=0$, corresponding to $G_{A B A}$-like inhibition. We also ran some simulations using a current approximation for synaptic input, with no apparent qualitative difference.

The Heaviside function reflects the fact that outputs are transmitted only when a cell's voltage exceeds its threshold. The external input is modeled independently for each cell as a Poisson process of pulses of width $t_{\text {spike, }}$ arriving at a mean rate $\lambda_{\text {ext }}$ (in $\mathrm{Hz}$ ) and of amplitude $V_{\theta} / N_{\text {con }}$. All inputs to a unit are scaled by the number $N_{\text {con }}$ of connections a unit makes with other units; the parameter $\alpha$ is of order $V_{\theta}$, here set without loss of generality to 1 . Furthermore, the input resistance in the update equation 3.1 is also set (without loss of generality) to 1. 
Equation 3.1 is supplemented by a reset mechanism to model spike generation. If $V_{i}(t)=V_{\theta}, V_{i}$ is reset to zero after a delay corresponding to the width of the action potential $t_{\text {spike, }}$ and kept at this value for a period $t_{\text {ref, }}$, representing an absolute refractory period. In our standard setup we have chosen, for simplicity, $t_{\text {delay }}=t_{\text {spike }}=t_{\text {ref }}=1 \mathrm{msec}$. Under these assumptions, equation 3.1 can be integrated over the characteristic delay time of $1 \mathrm{msec}$, resulting in a discrete approximation for the subthreshold domain:

$$
V(t+1)=[k V(t)+I(t)] \theta\left[V_{\theta}-V(t)\right]
$$

where $k=\exp (-1 / \tau)$ is the decay factor of the membrane potential.

This approximation assumes that the distribution of current at time scales smaller than $1 \mathrm{msec}$ will not change the dynamics, which is characterized by a time constant $\tau \gg 1$. In our standard model, $\tau=20 \mathrm{msec}$. It should be noted that the discretization equation 3.3 is natural in this context, since the original differential equation is not meant to capture the actual time course of the action potential during $t_{\text {spike }}$.

The reset after the spike is the simplest model of a rectifying potassium current. Since the unit needs time to recharge, the reset leads to an effective "refractory period." While it is true that the physiological refractory period is determined by the time course of a variety of voltage and calcium-dependent currents (Yamada et al. 1989), we are interested only in the temporal dynamics of spike times. When we henceforth refer to a refractory period in the model, we mean the effect of the reset.

3.2 Connectivity. As shown below, two aspects of the connectivity are crucial for high spike rate variability: locality of the connections and the range of inhibition. In the absence of local connections, for instance using all-to-all or sparse random connections, the population quickly reaches a steady or an oscillatory state (Amit and Tsodyks 1991a,b; Koch and Schuster 1992; van Vreeswijk and Abbott 1993; Tsodyks et al. 1993). Such states are characterized by low variability (except in the regime of very low firing rates).

With local excitation but no inhibition, waves of excitation originate from random centers on the lattice, but variability does not increase. If the excitatory coupling becomes too large, the activity in the network explodes, reaching a steady state with high activity, but low variability. The optimal connectivity pattern that leads to high variability consists of local excitation and inhibition (but with inhibition more distant than the excitation).

Our "standard model" was based on center-surround connectivity (see Fig. 2) on a rectangular array. Each unit is excitatorily connected to $N_{\text {con }}=50$ units chosen from a Gaussian probability distribution of $\sigma=2.5$ (in terms of the lattice constant), centered at the unit's position (square symbols in Fig. 2). The extent of excitatory connections was limited to a circular region of diameter 10 lattice units. Each unit was also connected 


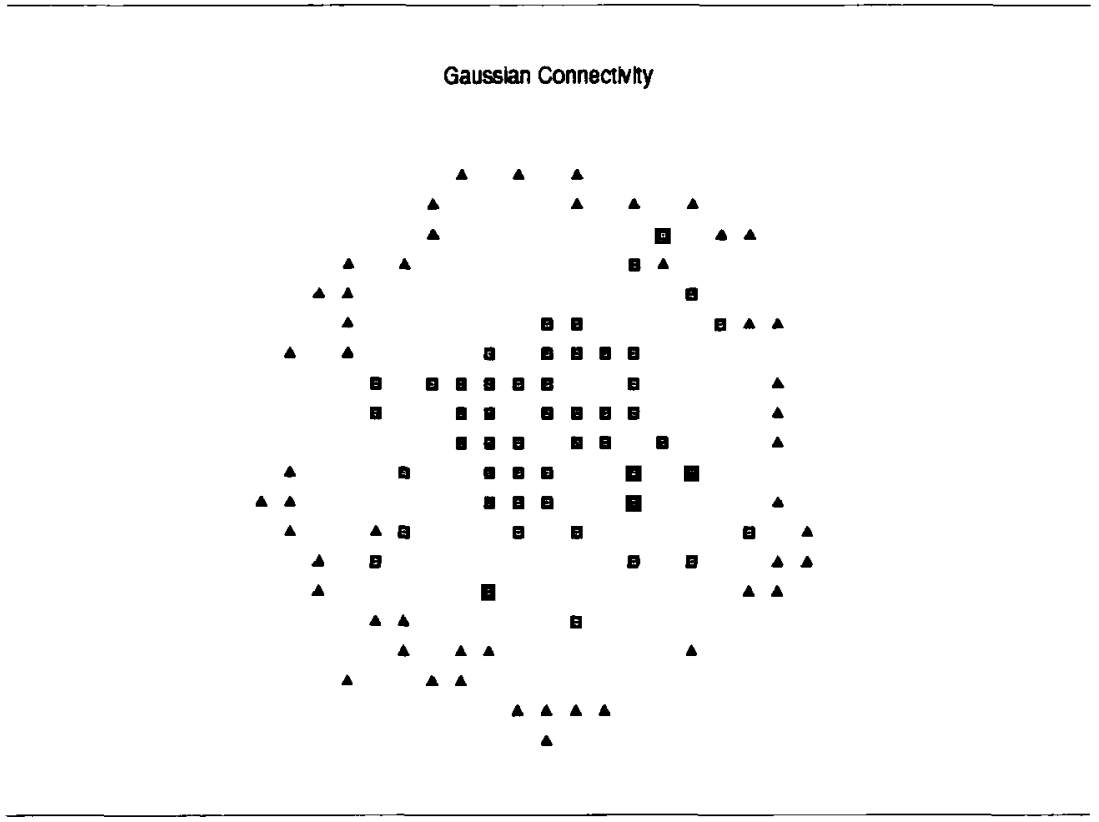

Figure 2: Basic gaussian connectivity pattern for the standard model. The cell (not shown) at the center of the rectangular array is connected in a probabilistic manner to units within a given distance determined by a gaussian distribution with $\sigma=2.5$ lattice constants. These short-range connections are excitatory (squares). The center cell also inhibits a fixed fraction of cells on an annulus 8 and 9 lattice constants away (triangles). During a particular simulation, the connectivity pattern is fixed, although the exact synaptic weight varies stochastically.

in an inhibitory manner to $N_{\text {con }}$ units chosen from a uniform probability distribution on a ring eight to nine lattice constants away (triangular symbols Fig. 2). No cell was allowed to make more than one connection to any other cell. Each cell's connections were generated independently at the start of the simulation and remained fixed thereafter, so that the geometric pattern of excitation and inhibition is not uniform across the lattice.

We occasionally use a sparse random connectivity. In this alternative setup, each unit makes $N_{\text {con }}$ excitatory and $N_{\text {con }}$ inhibitory connections to randomly chosen other units on the lattice, independent of distance.

The amplitude or weight of the excitatory connection is $f^{E}=\alpha / N_{\text {con }}$ and that of the inhibitory ones is $J^{I}=B J^{E}$ and is the same for all cells and synapses in the network. The normalization of the connection strengths by $1 / N_{\text {con }}$ in equation 3.2 takes into account the common physiological assumption that 50 to 100 summed EPSPs are needed to elicit a spike. For 
$\alpha \geq 1$ in the model, simultaneous firing of all local excitatorily connected units is sufficient to cause the receiving unit to spike.

To mimic the well-known stochastic character of synaptic transmission (Stevens 1993), we add an independent random offset to each synapse throughout the network at every iteration in equation 3.2. This corresponds to choosing the excitatory weight at each iteration from a uniform probability distribution $(\alpha-\Delta) / N_{\text {con }}<J^{E}<(\alpha+\Delta) / N_{\text {con }}$. Inhibitory weights were treated in the same fashion, but rescaled by $\beta$.

The possibility that a spike may fail to activate a synapse in an allor-none fashion, as often happens in slice preparations of cortical cells (Stevens 1993) was also investigated in control simulations. In this last case, spike transmission at any given synapse was likely to fail with a fixed probability $P_{f}$.

We used either cyclic wraparound or null-flux boundary conditions on a 100 by 100 unit lattice. In the latter case, activity beyond the boundary was treated as the mirror image of activity within the borders. Cyclic boundary conditions were preferred, since edge effects were absent.

3.3 The Standard Model. Unless specified otherwise, we always use in the following our "standard model" with gaussian center-surround connectivity on a 100 by 100 unit rectangular lattice with cyclic boundary conditions. Each cell excites $N_{\text {con }}=50$ excitatory and 50 inhibitory other cells and has a passive time-constant $\tau=20 \mathrm{msec}$. The Poisson distributed external input rate is $\lambda=2.3 \mathrm{kHz}$, while the excitatory weight $\alpha$ is drawn from the uniform distribution $[1.15,1.4]$. The inhibition has two-thirds the strength of excitation, that is, $\beta=0.67$ (EPSPs were renormalized by the magnitude of the driving potential from rest to make the IPSP and EPSP amplitude at the threshold voltage comparable). These parameters were chosen to achieve the maximal correspondence between model spike trains and those recorded from cells in monkey cortex.

We usually compare our standard model against spike trains from isolated units, that is, from units in a network with no lateral connections $(\alpha=0)$. To mimic the observed arrival of EPSPs and IPSPs, we used a combination of excitatory and inhibitory external Poisson input. The conductance change induced by each individual input, whether excitatory or inhibitory, was identical, but the rate of the inhibitory input was set to be 0.67 of the excitatory rate. Both inputs were in series with the appropriate synaptic battery, of $E_{\mathrm{exc}}=5 \times V_{\theta}=5$ and $V_{\text {inh }}=0$. To obtain approximately similar output rates, we had to increase the input rate to $15 \mathrm{kHz}$.

\section{Results}

We first discuss the overall dynamics of the entire network, before we turn toward properties of individual cells and, finally, of the local activity of small ensembles of neurons. 
4.1 Pattern Formation and Metastability. We investigated the dynamic behavior of the system under the influence of three parameters: the excitation coefficient $\alpha$, the ratio of synaptic inhibition to synaptic excitation $\beta=J^{I} / J^{E}$, and the rate of external input $\lambda$. High variability in the spike discharge results for $\alpha>V_{\theta}=1$, when the total excitation contributed by the generation of a spike, $N_{\text {con }}\left(\alpha / N_{\text {con }}\right)$, is larger than the decrease in potential caused by the resetting of the spiking neuron $V_{\theta}$. In particular, $1.2<\alpha<1.6$ and $0.5<\beta$ are optimal for obtaining high variability.

If the input rate $\lambda$ is high enough, ${ }^{3}$ one observes the emergence of clusters, or localized "hotspots" of neural activity (as in Fig. 3). The spatial range of inhibition effectively sets the bound on the radius of the clusters; for $N=50$ excitatory and inhibitory connections, the clusters' size is relatively insensitive to other parameter settings.

Within this radius, cells fire at a higher rate (due to recurrent excitation that is strongest at the center of the cluster and decreases toward the boundaries), while cells in regions between clusters are inhibited. For very high external input rates, the clusters become stable and merge into stripes or hexagonal patterns, while at intermediate rates, the system of clusters is metastable, as characterized by a high degree of mobility. In this regime, the behavior of the clusters is dominated by two conflicting forces: diffusion (cells at the edge send activation to cells in the cluster's vicinity) and the tendency to compactness, since the ring of inhibition acts as a "fire wall." As a result, the clusters fluctuate in size, move in a self-avoiding random walk-like fashion, and occasionally disintegrate or coalesce.

The formation of localized metastable patterns is illustrated in Figure 3 , where each frame displays the number of spikes emitted by all 10,000 units within the previous $50 \mathrm{msec}$. Regions of high activity do not remain fixed, but move from frame to frame. The motion of a typical cluster of high activity is shown in Figure 5. In some frames, elongated blobs/stripes appear and then disappear or change orientation in other frames. If one tracks the activity of individual fixed units during a long sequence of such frames, one observes large fluctuations leading to high variability in interspike intervals and total number of spikes.

More interestingly, the fluctuations take place not only on a scale of $50 \mathrm{msec}$, but on many time scales. Figure 4 shows a similar display, but using a 2-sec time window. Evidently even on this time-scale the fluctuations are not completely averaging out. However, on this extended time scale, the activation patterns show more elongated forms, suggesting some degree of averaging over the patterns' trajectories.

For low inhibition values, $\beta<0.33$, one obtains traveling waves (long fronts of activity) that are not fully periodic. Under such conditions the

\footnotetext{
${ }^{3}$ Empirically, the asymptotic subthreshold voltage $V=\lambda \tau / N_{\text {con }}$ must be at least $0.6 V_{\theta}$.
} 

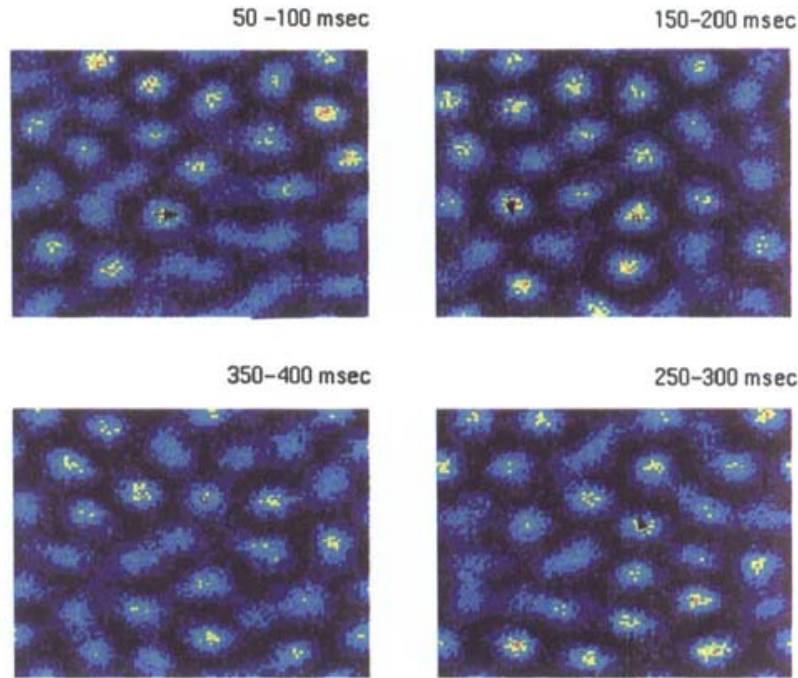

Figure 3.
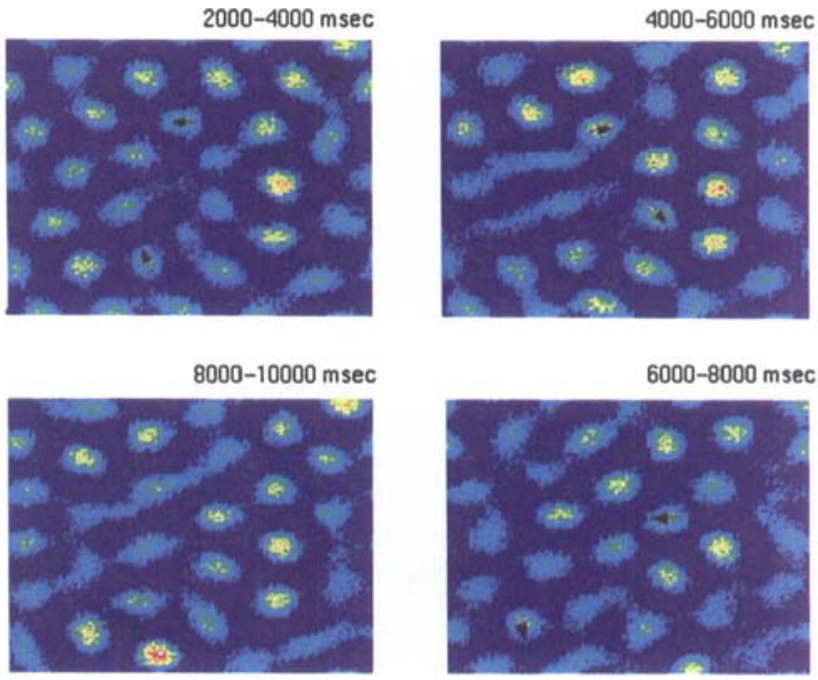

Figure 4 . 
individual cells show low $C_{V}$ values. This parameter regime will not be further discussed in this work.

\subsection{Single Cell Properties.}

4.2.1 Interspike Interval Variability. We computed the coefficient of variation $C_{V}$-without attempting to renormalize $C_{V}$ for the existence of a refractory period-in our population of 10,000 cells over a 400 -sec long simulation using a stationary input frequency of $\lambda=2.3 \mathrm{kHz}$ to all cells in the standard model (when the initial $10 \mathrm{sec}$ following the onset of the stimulus were eliminated, no noticeable difference resulted). The resulting values of $C_{V}$ for individual cells are displayed in Figure 6 as a function of the mean interspike interval. Higher $\lambda s$ (or weaker inhibition) give rise to higher spiking frequencies and lower interspike intervals, while maintaining high variability.

Note that almost all values of $C_{V}$ are on the order of one or larger. The pattern of observed $C_{V} s$ reproduces qualitatively the $C_{V}$ values measured for cells in cortical areas V1 and MT in the awake monkey responding to bars and to clouds of moving dots (Softky and Koch 1993). As discussed above, this is surprising given that, for an integrate-and-fire model at high output rates (when the effect of the membrane leak can be neglected), $C_{V}=1 / \sqrt{N_{\theta}}$. For our parameter range, $N_{\theta} \approx 50$ and therefore $C_{V}=0.14$.

We computed the $C_{V}$ of the cells without any lateral connections in the network, that is, when the cells are only responding to the external input (lower cloud of dots in Fig. 6) and find that the $C_{V}$ values are on average

Figure 3: Facing page. Spontaneous symmetry breaking in neural pattern formation. Each frame represents the summed activity over $50 \mathrm{msec}$ in a simulation for random external input. These "snapshots" of neural activity are shown at $100 \mathrm{msec}$ intervals from each other in a clockwise arrangement starting with the frame in the upper left-hand corner. Lighter colors denote higher firing rates (maximum firing rate $120 \mathrm{~Hz}$ ). The direction of motion for selected clusters is shown by an arrow; the position of the moving cluster in the next frame is indicated by a diamond shape. Parameters for this and all subsequent figures pertaining to the model were external Poisson input rate $\lambda=2.3 \mathrm{kHz}$, connection strength $\alpha$ is uniformly distributed between 1.15 and 1.4 , membrane time constant $\tau=20 \mathrm{msec}$. Inhibition equals $2 / 3$ of that of excitation $(\beta=0.67)$.

Figure 4: Facing page. Here each frame represents the summed neuronal activity within a 2-sec long time window in the presence of random external input. All other parameters are as in the previous figure. The self-similarity of the neuronal activity across different time scales is evident in the emergence of clusters at the $50 \mathrm{msec}$ as well as at the 2-sec time scale. The highest number of spikes in these frames for any unit is 145 , i.e., $72 \mathrm{~Hz}$. 


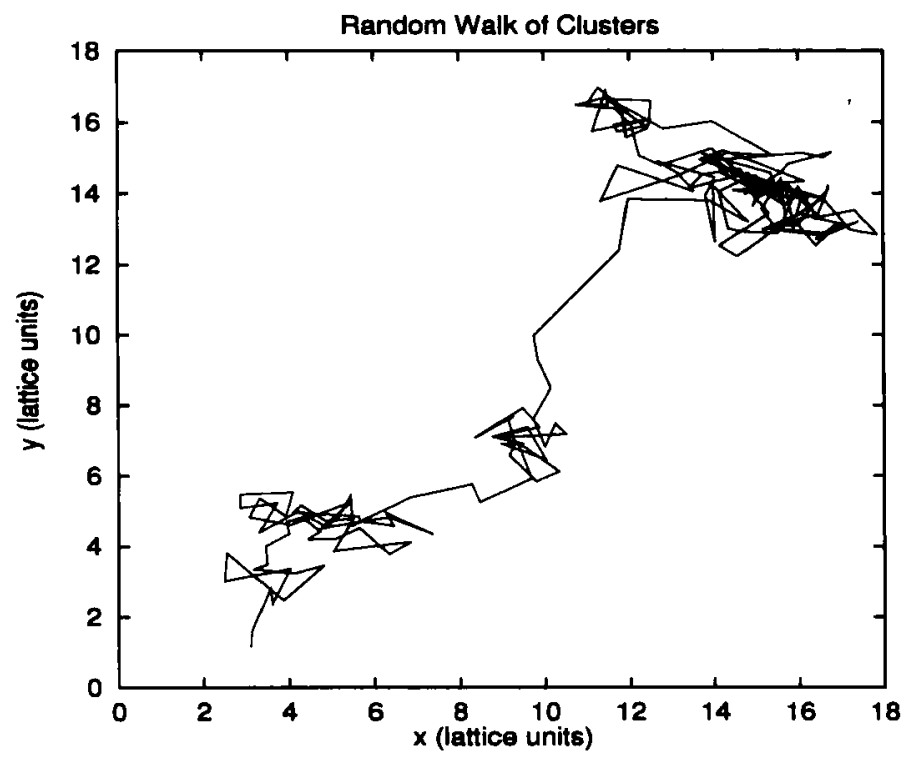

Figure 5: To illustrate the motion of a typical cluster seen in Figure 3, the center of a cluster is tracked over $10 \mathrm{sec}$ of simulation. Each vertex in the graph represents the cluster's position averaged over $50 \mathrm{msec}$. Repulsive interactions with surrounding clusters generally constrain the motion to remain within a certain radius. This vibratory motion of a cluster is occasionally punctuated by longer-range diffusion.

reduced by a factor of two. Qualitatively, a similar reduction by a factor of three in $C_{V}$ occurs in a network with sparse nonlocal connections as discussed in the "Model" section. At the same mean spiking frequency, that is, same mean time between spikes, the isolated and the nonlocal networks show much lower variability than the network with the centersurround connectivity.

4.2.2 Interspike Interval Histogram and Power Spectra. The power spectra of spike trains from individual units in our standard model (Fig. 7) are similar to those published in the literature for nonbursting cells in area MT in the behaving monkey (Bair et al. 1994). Power spectra were generally flat for all frequencies above $100 \mathrm{~Hz}$. The effective refractory period introduces a dip at low frequencies (Bair et al. 1994). Given the long duration of individual spike trains, here $400 \mathrm{sec}$, the frequency resolution is high enough to observe the $1 / f^{0.8}$ decay at low frequencies (Fig. 7). 


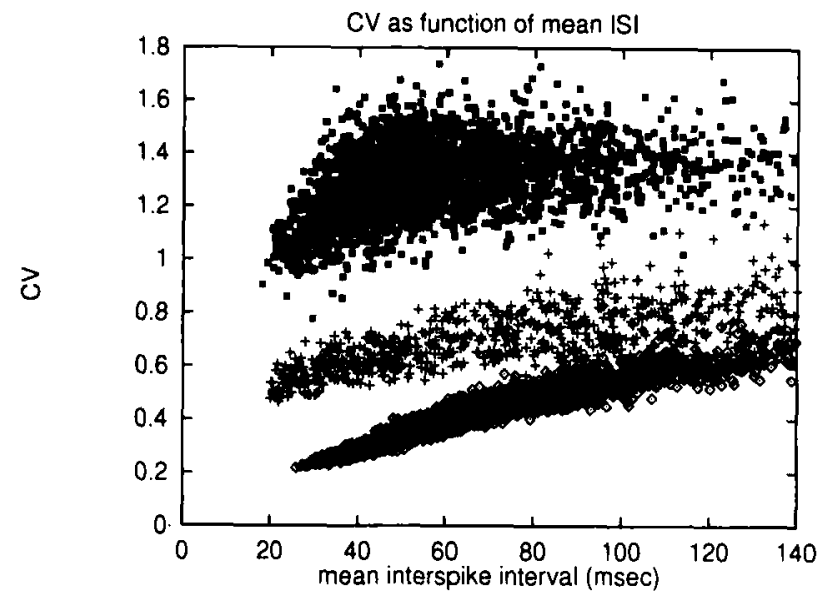

Figure 6: Coefficient of variability, $C_{V}$, of a representative range of cells shown against the average time between spikes, that is, the inverse of the mean firing rate. The solid dots in the upper cloud are from our Standard Model. The crosses in the middle cloud represent the behavior when all network effects are eliminated (i.e., $\alpha=0$ ), and units receive both an excitatory and an inhibitory stream of external Poisson input, with $\lambda_{i}=0.67 \times \lambda_{e}$. Without inhibition, the associated $C_{V}$ values are reduced by a factor of about 2 . The diamonds in the lowest cloud are from cells in a random network with sparse, nonlocal connections that have no organized topography. The same number of inhibitory and excitatory connections, 50, were used as in the local center-surround connection scheme. All connections are reciprocal but otherwise random. Parameters for this run were otherwise the same as for the standard model.

Since Bair et al. used a frequency resolution of $4 \mathrm{~Hz}$ (instead of $0.25 \mathrm{~Hz}$ for Fig. 7), they could not have observed a $1 / f$ component of the type shown for the model, even if $1 / f$ noise had been present in the real data.

Notice that the power spectrum associated with a pure Poisson process of rate $\lambda$ is flat at all frequencies (no particular frequency is preferred), except for a delta function peak at the origin:

$$
S(f)=\lambda+2 \pi \lambda^{2} \delta(f)
$$

If the point process is Poisson with an absolute refractory period drawn from a gaussian distribution of temporal width $\sigma$, the power spectrum 

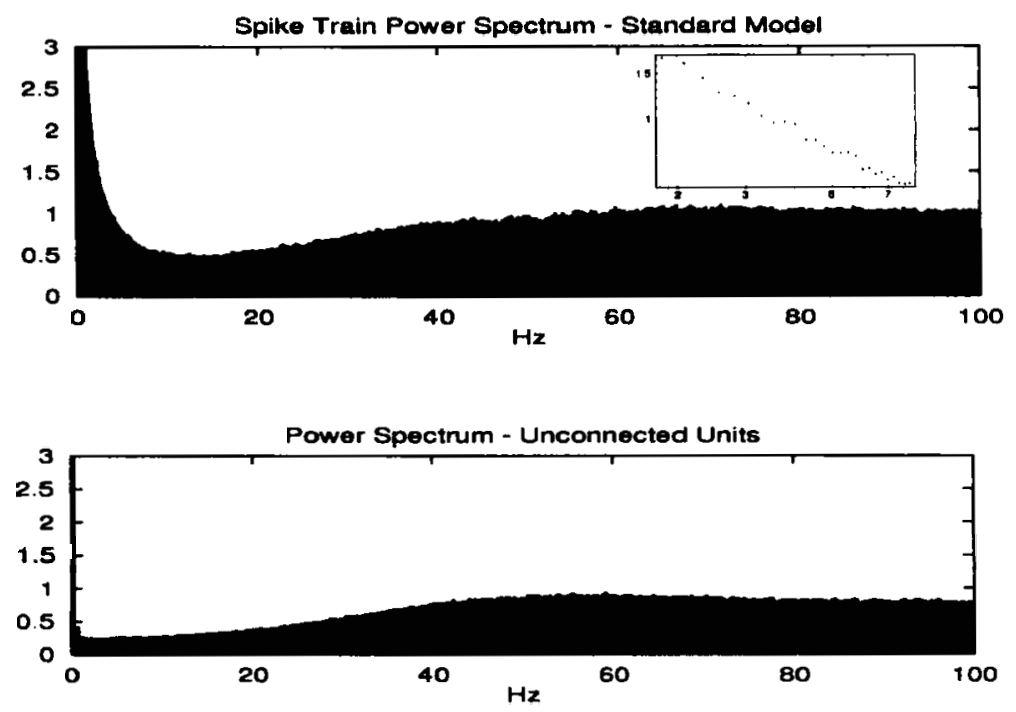

Figure 7: Power spectra associated with the spiking activity of single units. The spectra for 19 units were computed individually from a 400-sec long run for the standard model and averaged in the uppermost spectrum. The average spiking frequency of the cell throughout the run is $18.1 \mathrm{~Hz}$. At low frequencies, the power spectrum behaves as $f^{-0.8 \pm 0.017}$ up to a cut-off frequency of $\approx 8 \mathrm{~Hz}$ (see superimposed solid line, and inset, which displays the same graph on a $\log -\log$ scale). For comparison, the lower spectrum represents the behavior for the same parameters, but without any lateral connections. Note the absence of $1 / f$ noise here. To obtain reliable estimates of the spectrum's low-frequency components, very long spike trains are required.

develops a dip at low frequencies:

$$
S(f)=\lambda\left[1-\sqrt{2 \pi} \lambda \sigma e^{-2(\pi f \sigma)^{2}}\right]
$$

with $\lambda \leq 1 /(\sqrt{2 \pi} \sigma)$ (Bair et al. 1994).

Figure 7 also shows the power spectrum in the disconnected network, that is, when $\alpha=0$. Individual units only receive external input. The major difference to the spectrum in Figure 7 is the lack of a $1 / f$ dependency around zero. Note the very weak peak around $50 \mathrm{~Hz}$ due to the more regular firing pattern.

The ISI histogram of the standard model, averaged over 19 units, is shown in Figure 8. Because of the effective refractory period, interspike intervals lower than $4 \mathrm{msec}$ are not observed, while the clustering nature of the model leads to occasional long intervals. The slow decay in the 


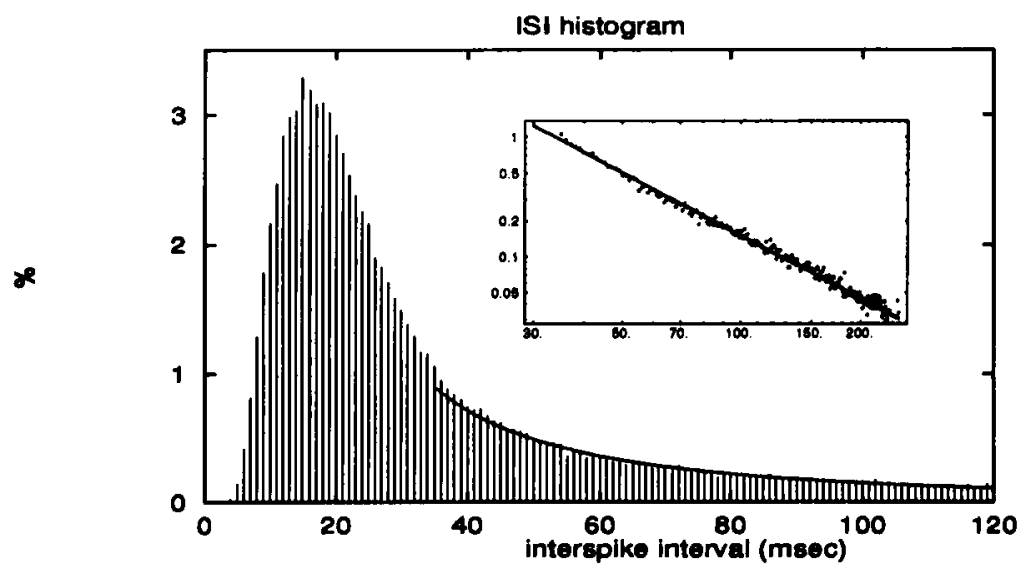

Figure 8: Interspike interval (ISI) histogram of single unit activity averaged over 19 units in the standard model. The inset displays the same graph on a $\log -\log$ scale. The best fit to the power-law decay exponent of the ISI histogram between 25 and $300 \mathrm{msec}$ is $-1.70 \pm 0.02$, implying a fractal dimension of the underlying point process of 0.7 . The tail of the ISI histogram for isolated units (i.e., for $\alpha=0$; not shown) decays exponentially.

ISI histogram for long intervals has an associated power-law exponent in the trailing edge of the histogram of 1.7 over one decade (from 25 to $300 \mathrm{msec}$ ). The corresponding fractal covering dimension $D$ is 0.7 (equation 2.7). In contrast, the ISI for units in the disconnected network ( $\alpha=0$, not shown), has a tail that decays exponentially, with $\tau=15.8 \mathrm{msec}$.

4.3 Local Fluctuations and the Field Potential. To show the fluctuations in the input to individual units, we display in Figure 9 the total excitatory input received by a single cell from other cells in the population (excluding $I^{\text {ext }}$ ). The lateral excitatory input to a cell is equivalent to the total activity in an area of radius 5 covered by excitation.

The power spectrum of this input signal (Fig. 10) has a small peak at about $40 \mathrm{~Hz}$ (see the inset in Fig. 10) due to the internal dynamics of hotspots that oscillate in size. Increasing the area over which the total activity is measured to a disk with a radius of 9 lattice units leads to an increase in the periodicity, as observed by the enhancement of the $40-$ $70 \mathrm{~Hz}$ component and the disappearance of the $1 / f$ components around zero (Fig. 11). The existence of a peak in the power spectrum of the ensemble activity is robust to rescaling the size of clusters (by changing the number of connections), the size of the network, the addition of noise, 


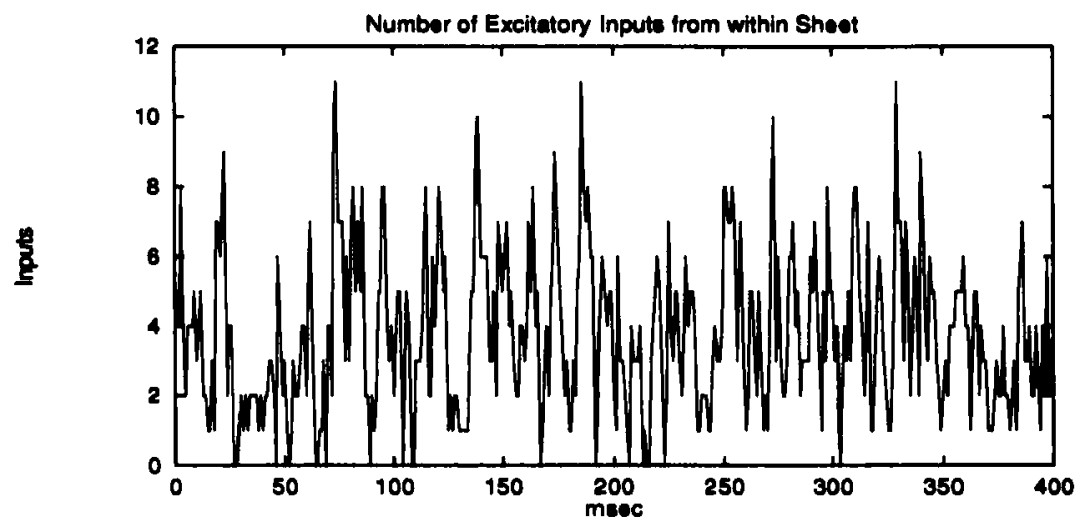

Figure 9: The total number of excitatory inputs into one particular neuron as a function of time. Given our connection geometry, this input comes from cells within 5 units distance. The external input is not included here and, for our standard input rate of $\lambda=2.3 \mathrm{kHz}$, corresponds to a mean input level of 1.8 lateral inputs per msec. Thus, local feedback connections dominate the network, in agreement with the canonical microcircuit hypothesis (Douglas and Martin 1990). Note the quasiperiodicity around $25 \mathrm{msec}$ in the strength of this signal, which arises from the internal dynamics of a cluster (see Fig. 10).

and to the introduction of time-varying synaptic inputs (here, decaying exponentials).

Since there are no (excitatory) long-range connections to link clusters, different clusters oscillate in size independently with different phases. The activity over the entire network is thus the sum of $n$ incoherent quasiperiodic oscillators, where $n$ is the average number of clusters on the lattice.

4.3.1 Cross-Correlation Among Cells. Other well known measures of spike train analysis are the auto and cross-correlation functions. In Figure 12 we display some typical correlation functions obtained for spike trains from the same 400-sec long simulation as in Figure 7.

The correlation functions were computed according to

$$
C\left(t^{\prime}\right)=\frac{T}{T-t^{\prime}} \sum_{t=1}^{T-t^{\prime}}\left[x_{i}(t) x_{j}\left(t+t^{\prime}\right)\right]
$$

where $x_{i}$ and $x_{j}$ represent two spike trains (with either no or one spike event per msec bin), and $T$ is the total duration of the recorded train. The multiplicative factor $T /\left(T-t^{\prime}\right)$ is necessary for normalization, because we 


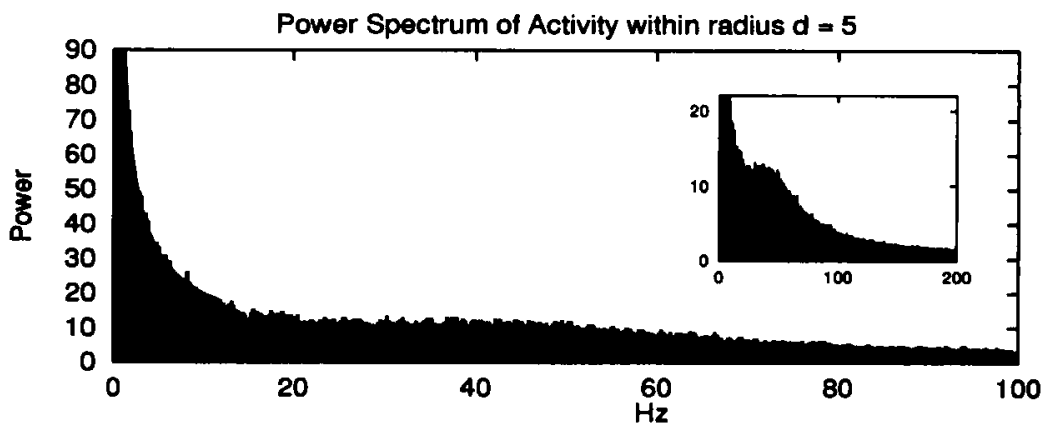

Figure 10: Power spectrum of the summed spiking activity over a circular area of radius 5 recorded from a fixed point on the lattice for $400 \mathrm{sec}$. This spectrum is roughly equivalent to the power spectrum of the lateral excitatory input (see Fig. 9), since short-distance connections are solely excitatory. At low frequencies, $S(f) \propto f^{-\nu}$, where $\nu=0.69 \Omega 0.02$ (see the solid line). The inset shows an enlarged part of the spectrum, revealing a small peak around $40 \mathrm{~Hz}$.

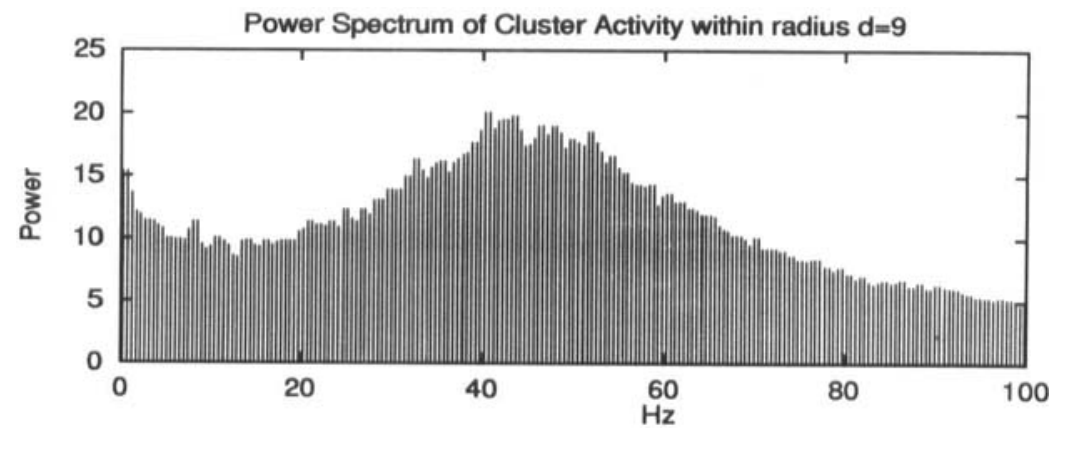

Figure 11: Power spectrum of the summed spiking activity over a circular area the size of a single cluster (with a radius of 9 lattice constants) recorded from a fixed point on the lattice for $400 \mathrm{sec}$. The signal is the total number of units spiking at any given iteration. Compared to the power spectrum of the spiking activity averaged over 5 units (previous figure), the peak, at $43 \mathrm{~Hz}$, becomes much more noticeable, while the $1 / f$ component disappears. Similar power spectra were obtained for the total activity on small lattices ( 20 by 20$)$ that contained only a single cluster and for interacting clusters whose positions were tracked on the full-scale lattice ( 100 by 100$)$. 

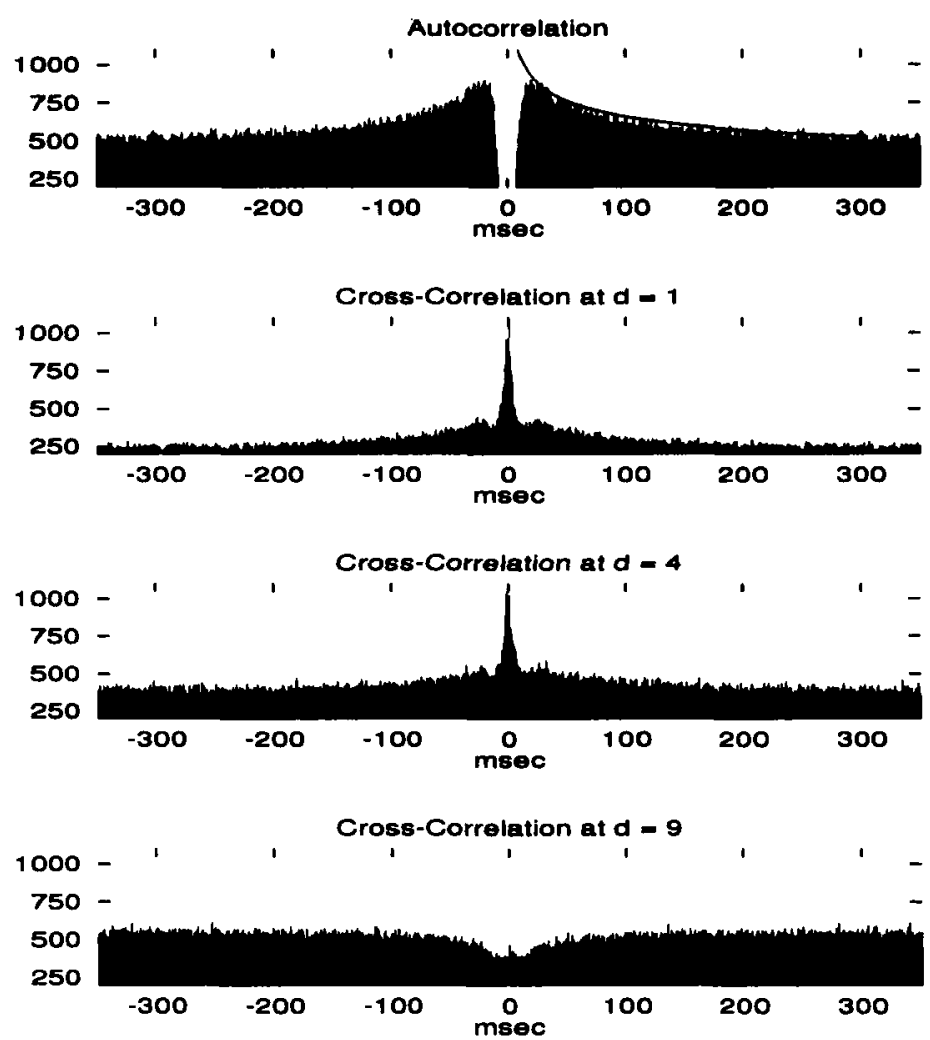

Figure 12: Auto- and cross-correlation functions between cells separated by $d$ units in the standard model. Each graph represents the average correlation functions over $400 \mathrm{sec}$ of four pairs of cells that were randomly chosen (the baselines, therefore, are different). The autocorrelation $A(t)$ behaves as $1 / t^{0.21}$, consistent with a power spectrum that behaves as $1 / f^{0.8}$. The graph of the best power-law fit to the autocorrelation is shown (numerical goodness of fit $\pm 0.004)$, raised slightly for reasons of legibility. Cross-correlograms similar to these have been observed in cat visual cortex (Nelson et al. 1992).

use finite trains to compute the correlations. When the two spike trains are identical, $i=j$, the result is the autocorrelation function $A(t)$.

The autocorrelation (Fig. 12a) shows the effect of a refractory period, followed by a period of enhanced firing probability which decays slowly to an asymptotic value, via a power law with exponent -0.21 . This behavior takes place up to a temporal cutoff of $300 \mathrm{msec}$, after which 
the autocorrelation function reaches the baseline of chance coincidence. We should note that a power law decay is not the only possible model that will fit the data. The power law decay in the autocorrelation spans roughly one decade, which is not long enough to rule out the hypothesis of an exponential decay. The difference between the best power law fit $a t^{-b}$ and the best exponential fit $a \exp (-t / \tau)+c$ does not reach the level of statistical significance, as based on a $\chi^{2}$ test assuming Poisson-distributed errors.

In Figure $12 b, c, d$ we display cross-correlation functions for cells at three different spatial separations. The excitatory cross-correlations exhibit three main features: a sharp central peak, termed a "castle" in the neurophysiological literature (Nelson et al. 1992), flanked by small secondary peaks, and a slow decline to an asymptotic value, termed "hill" by (Nelson et al. 1992), characterized by the same power exponent as for the autocorrelation (i.e., -0.21 ). The cross-correlation for $d=9$ shows a central dip with a slow recovery (governed by the same exponent) to the asymptotic level. This dip around the origin is caused by the action of inhibition located 8 to 9 units away (see Fig. 2).

4.3.2 Variability and Fractal Firing Patterns. Figures 3 and 4 illustrate that the activity of the neural population undergoes fluctuations on several time scales. Self-affine correlated fluctuations occur on all scales between 20 and $300 \mathrm{msec}$, based on the power law behavior of the autoand cross-correlations, as well as that of the ISI distribution over this range. To obtain a quantitative measure of these fluctuations, we calculated the variance-mean curve by dividing a long simulated spike train into nonoverlapping periods of time $T$, and then computing the mean and variance in the number of events in intervals ranging from 20 to $5000 \mathrm{msec}$.

The results are displayed in Figure 13 on a logarithmic plot. The slope of the curve in Figure 13 is $1.402 \pm 0.007$ between $N=0.5$ and 20 , corresponding roughly to intervals between 20 and $800 \mathrm{msec}$ (at an average rate of $23 \mathrm{~Hz}$ ). For $t>1 \mathrm{sec}$, temporal correlations in the spike train have decayed to chance level, so the variance in $N$ behaves once again in a Poisson manner, that is, the slope of the standard model is equal to the slope of the Poisson process. For times shorter than the minimum spike interval, $\operatorname{Var}(N)=N$, since there can be at most one spike in these intervals.

We also simulated the second experimental paradigm that evaluates the variability in spiking in which many trials of fixed duration were repeated. For this we performed a set of 25 simulations of $2.2 \mathrm{sec}$ each (but only used the last $2 \mathrm{sec}$ ), for each afferent input level (changing the initial conditions and the random noise fluctuations, but keeping the connectivity pattern the same), and computed the mean number of spikes $N$ and variance $\operatorname{Var}(N)$ for 60 randomly chosen cells in the network. The 


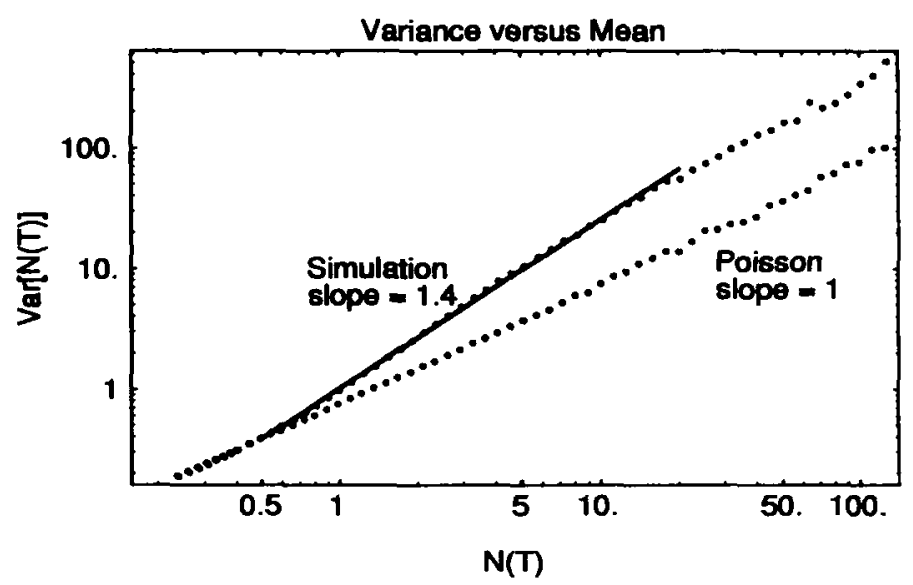

Figure 13: Log-log plot illustrating how the variance in the number of spikes varies as the mean number of spikes $N$ for variable sampling intervals $T$. The exponent in the power law $\operatorname{Var}(N) \propto N^{\nu}$ is $\nu=1.4$ for the standard set of parameter values. For comparison purposes, results from a Poisson train with refractory period $5 \mathrm{msec}$ are superimposed, showing a slope of 1 . The base firing rate for both processes is roughly $23 \mathrm{~Hz}$. Self-affine fractal behavior of the spike train extends over the range from 20 to $800 \mathrm{msec}$ and is evident in the fact that the variance increases faster than the mean. For $t>1 \mathrm{sec}$, temporal correlations in the spike train have decayed to chance level, so the variance in $N$ behaves again in a Poisson manner (as discussed in Section 2.2). Typical power law exponents $\nu$ for real spike trains from the peripheral auditory nerve (Teich 1989) and parietal cortex (unpublished data) range from 1.2 to 1.7 for spontaneous activity.

procedure was repeated with different values of stimulus strength to extend the range of the mean number of spikes.

These data points, plotted on a log-log scale, are shown in Figure 14. The best linear fit (see solid line) has a slope of $1.54 \pm 0.02$, higher than the slope in the previous figure using a different paradigm. An average slope of 1.21 and 1.10 was measured in similar experiments carried out in cells in cortical areas V1 and MT, respectively, in the monkey responding to random dots (Snowden et al. 1992). Softky and Koch (1993) find the slope for MT to be 1.25. For comparison, we also plotted the response of 60 isolated units, that is, with $\alpha=0$, subject to the same stimulation protocol. Here the slope of the best linear fit is $0.436 \pm 0.004$. 


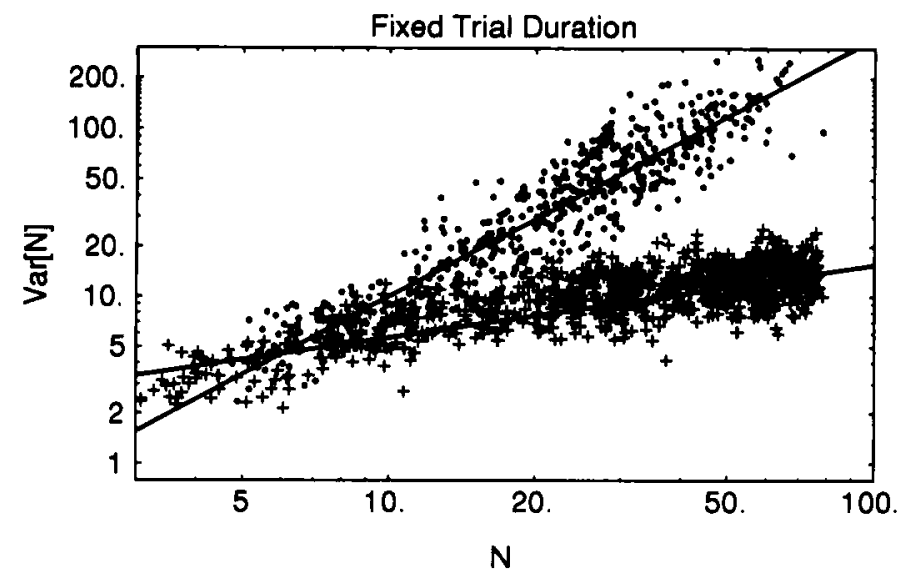

Figure 14: Log-log plot illustrating how the variance in the number of spikes $\operatorname{Var}(N)$ varies as the mean number of spikes $N$ for a fixed sampling interval $T$. Spike trains from 60 randomly chosen cells in a 56 by 56 size network were recorded. The external input rates were ramped from 1.9 to $2.3 \mathrm{kHz}$ in increments of $50 \mathrm{~Hz}$, with 25 trials at each stimulus level lasting $2.2 \mathrm{sec}$. The last $2 \mathrm{sec}$ of each trial were used for this analysis. The slope of the best linear fit on the log-log plot is $1.54 \pm 0.02$ (filled circles). The initial slope for the first four stimulus levels $(1.9-2.05 \mathrm{kHz})$ is lower: $1.36 \pm 0.04$. Similar plots for cells in visual cortices $\mathrm{V} 1$ and $\mathrm{MT}$ responding to moving bars and random dots show slopes of around 1.2 (Snowden et al. 1992). We also plotted the same data for 60 units in a disconnected network, i.e., $\alpha=0$ (crosses). Here, the best linear fit is $0.436 \pm 0.004$.

\section{Discussion}

Our goal has been to forge a theoretical link between the statistics of spike trains from single cells and the dynamics of the entire network. Furthermore, we want to propose a solution to the dilemma posed by Softky and Koch (1992, 1993), on how to obtain high variability in networks of rapidly firing neurons that integrate over large number of synaptic inputs. Our approach here is to simulate a simple network of spiking cells receiving external input, reminiscent of a cortex receiving input from a dynamic random dot display, and to relate our findings to experimental findings in the cortex of cat and monkeys. Although Softky and Koch did offer a solution to the problem of high variability-neurons that act like coincidence detectors (see also Abeles 1982, 1991) —we here offer an- 
other solution more in line with standard physiological thinking about the biophysics of pyramidal cells.

Our simple network of integrate-and-fire units not only achieves high variability, but also shows several other noteworthy features that are shared with experimental data from cat and monkey visual cortex: the power spectrum of the local-field potential frequently shows a peak around $40 \mathrm{~Hz}$ while the spectrum of single unit recordings very often does not (in particular in the primate), the cross-correlation has a typical castle-upon-hill structure and the ratio of variance to the mean of the number of spikes increases faster than expected of a Poisson process. Various measures of the spiking dynamics in our network shows selfsimilar (fractal) behavior. It is at present not known to what extent such scaling laws can be found in cortical cells. Before we go on, let us discuss the fundamental limitations and assumptions of our modeling effort.

5.1 Limitations and Assumptions of Our Model. Our model of a single cell is a leaky integrate-and-fire unit receiving conductance inputs (Knight 1972). One could argue that such cells do not show the temporal dynamics of cells with a variety of voltage- and time-dependent Hodgkin-Huxley like currents acting over different time-scales. However, our previous research (Softky and Koch 1993; Bernander et al. 1994) has provided ample evidence that an anatomically very detailed compartmental model of a neocortical pyramidal cell with seven voltage-dependent currents at the cell body and a passive dendritic tree has temporal dynamics very similar to those of a leaky integrate-and-fire unit.

The fundamental assumption critical to the functioning of the model is the center-surround pattern of connectivity, with short-range excitation and a longer-range inhibition. Note that this constraint must only hold on average. If both excitation and inhibition are short-range, we fail to reproduce the observed spike statistics such as power spectra and ISI histograms, since the lattice dynamics are now dominated by waves of neural activation. The resulting spike trains have much lower $C_{V}$ values.

Our model is consistent with the "canonical microcircuit" hypothesis (Douglas and Martin 1990) in which massive excitatory recurrent feedback dominates the behavior of cortex: for our standard model with an input rate of $2.3 \mathrm{kHz}$ (see Section 3.3), the average sum of lateral currents is at least $50 \%$ larger than the external, sensory driven, current. Examining Figure 9 reveals that for short periods the lateral excitatory current greatly exceeds the external current at a $2.3 \mathrm{kHz}$ input frequency by an even greater margin. Higher input rates lead to higher amplification factors. The circuit in the model thus serves to amplify the afferent input signal, particularly locally through the formation of "hotspots" (clusters).

While anatomical and physiological evidence does not provide an unequivocal support for this connection scheme, some physiological evidence for the validity of our assumption can be found. Hess and coworkers (Hess et al. 1975) found that iontophoretic application of the 
excitatory agonist glutamate to visual cortex of anesthetized cats induces excitation of neurons within $100 \mu \mathrm{m}$ of the application site and distant inhibition at distances between 100 and $500 \mu \mathrm{m}$. Similar studies in rat somatosensory cortical slice preparations (Silva and Connors 1987) confirm the general pattern of an inhibitory surround enclosing the excited region for all layers of cortex with one exception: layer IV, which receives direct afferent sensory input.

A second critical assumption for our model is the existence of fast inhibition. Simulations performed with exponentially decaying synaptic inputs show that the cluster scenario fails if the inhibitory synapses are much slower than the excitatory synapses. When, for instance, the inhibitory synapses are five times slower, the inhibitory "fire wall" comes into play too late, after the excitation has already spread outside the cluster's domain. In this case the population is entrained into periodic oscillations, which radically changes the ISI histogram of single cells.

Microstimulation studies give us an estimate of the speed with which inhibitory effects take hold: Asanuma and Rosen (1973) showed that inhibition outdistances excitation in motor cortex, with the onset of all excitatory and inhibitory interactions occurring within $3 \mathrm{msec}$ of stimulation.

Although inhibition must act through interneurons, the inhibitory response to stimulation can be almost as fast as the excitatory response in cortex. Also, spike-triggered averaging of EPSPs and IPSPs elicited synaptically in slice preparations show identical rise times for IPSPs and EPSPs of $\approx 1.5 \mathrm{msec}$ (Komatsu et al. 1988). We should notice, however, that the coexistence of long-term and short-term inhibition is not ruled out; in fact, a slower $\mathrm{GABA}_{B}$ type current in the model would lead to greater mobility of the clusters of excitation and thus to greater variability.

At the moment, our model does not contain any explicit interneurons, that is, individual units can both excite one set of postsynaptic targets while simultaneously inhibiting another set. Our future work will do away with this unrealistic feature of our model, but at a price of increased number of neurons and therefore an increased computation time. This constraint also held the connectivity to $N_{\text {con }}=50$, a small fraction of the divergence seen in cortex. The connectivity scheme Figure 2 is meant to approximate a more realistic connectivity where both the inhibition and excitation are probabilistically spread over broader areas. To investigate the effect of rescaling the model in a preliminary manner, $N_{\text {con }}=150$ connections per neuron (instead of 50) were used in short control runs, yielding similar results to the $N_{\text {con }}=50$ case. $^{4}$

We did not attempt to model cells that burst, that is, that discharge 2-4 spikes within $10 \mathrm{msec}$ or less. A large fraction of cortical cells responding to sensory events in the awake monkey frequently fires bursts (Werner

\footnotetext{
${ }^{4}$ Although larger $N_{\text {con }}$ lead to more stable (thus less variable) firing patterns, "metastability" is restored once a synaptic "failure" process is taken into account, even for very low failure probabilities $P_{f}<5 \%$. See Section 3.
} 
and Mountcastle 1963; Bair et al. 1994). It is easy for bursting cells, with more complicated internal dynamics, to show arbitrarily high values of $C_{V}$. Instead, we here assume the standard reset mechanism of integrateand-fire neurons; therefore, short interspike intervals were rare.

What we consider the most important limitation of our current study is that we assumed stationary and uncorrelated input to all cells of the network. In particular, we did not model the bars or gratings that are the most common form of visual stimulus of the various experimental studies we cite. However, we were surprised by the rich dynamic behavior and its qualitative relationship to experimental data. Yet, this present study is clearly but a starting point for more realistic and sophisticated models of cortical networks.

In the following, the main results of the model will be discussed.

5.2 Pattern Formation. We have shown that "hotspots," that is, patterns of excitation, emerge even for homogeneous Poisson input to our network (Figs. 3 and 4). We carried out similar simulations using completely isotropic, circular connectivity patterns with fixed synaptic weights and again observed the emergence of circular excitation patterns (not shown). In our network, this pattern formation process implies spontaneous symmetry breaking due to random spatial correlations in the external input.

The emergence of hexagonal and stripe-like patterns through symmetry-breaking in continuous firing-rate models with similar connectivity has been previously demonstrated (Cowan 1982). In our model, however, the patterns of excitation are not stable; rather, they are subject to a stochastic diffusive process, leading to spatiotemporal fluctuations on many time scales (Fig. 3).

It should be possible to directly visualize these moving "hotspots" using high-resolution optical imaging of the intact cortex in primates based on voltage-dependent dyes or intrinsic signals (Grinvald 1992). Indeed, moving circular or elliptic regions of high neuronal activity have been reported in visual cortex of the anesthetized squirrel monkey and the rat using voltage-sensitive dyes in response to electrode stimulation. Orbach et al. (1992) observed motions of such clusters on the millisecond time scale; multiple clusters were sometimes elicited in response to stimulation at a single point, and, in one instance, a single cluster was seen to split into two separate centers of high activity.

5.3 High Interspike Variability. We have shown that neural spike trains from our standard model have large coefficients of variation, that is, $C_{V}$ values between 1 and 1.5 for rates up to $50 \mathrm{~Hz}$ (Fig. 6). Increasing the external Poisson input rate or lowering $\beta$, the ratio of inhibition to excitation, results in higher firing rates, which we did not explore here. 
These large $C_{V}$ values are reflected in the slow decay in the tail of the ISI (Fig. 8), here as $1 / t^{1.7}$. This is in sharp contrast to the full ISI for units in the disconnected network that decay exponentially. We do not know at present whether such power-law decay in the ISI has been seen in cortical cells, but are studying this issue systematically.

In their study of $C_{V}$ from nonbursting cells in cortical areas V1 and MT, Softky and Koch (1993) observed equally high values. The $C_{V}$ associated with the disconnected units receiving only excitatory and inhibitory external Poisson input is about a factor of two lower (Fig. 6). Without the inhibitory Poisson input, the associated $C_{V}$ would be further reduced by a factor of $\sqrt{5}$ (see equation 2.4). $C_{V}$ values are even further reduced in a network with random connectivity.

In our model, the high degree of randomness observed in the single unit firing statistics arises from the amplification of correlated fluctuations, resulting in clusters of excitation moving through the cortical layer. This can be compared to a recent model of short-term memory that was proposed to account for the observed interspike interval histogram of spikes in IT cortex of behaving monkeys (Zipser et al. 1993). Intrinsic variability in this model is the result of stochastic transitions between two firing rates (attractors in the language of dynamical systems) inside a probabilistic network of McCullough-Pitts neurons. Our model provides an actual mechanism for such transitions while generalizing the approach of Zipser and colleagues to include the leak of the membrane time constant and noise in the form of fluctuating Poisson external input to the cells. To be precise, the model has not only two fundamental firing rates, but rather a continuum of rates related to the cell's position relative to the cluster centers; moreover, as opposed to Zipser et al. (1993), who fitted interspike interval distributions with two exponentials of different time constants, in our model the interval distribution and correlation functions decay with a power law. Consequently, we find that the firing pattern is fractal up to a time scale of about $800 \mathrm{msec}$.

It should be noted, however, that a power law decay can arise as the superposition of many exponentials with different time constants. If the majority of the input EPSPs is distributed as an infinite-range power law $1 / t^{\alpha}$ with $\alpha<3$, the central limit theorem does not apply to the sum of EPSPs since the variance of the distribution is not finite. Consequently, for finite-range power laws, the $C_{V}$ of a sum of $N_{\theta}$ pulses will decrease (initially) more slowly than $1 / \sqrt{N_{\theta}}$ as the number of pulses increaseseven the sum of a large number of pulses can still have a high $C_{V}$.

As we mentioned previously, alternative solutions to the variability problem are either very large hyperpolarizing (inhibitory) currents or an active dendritic mechanism for coincidence detection at the millisecond level. To achieve the same high variability as in the model based on concurrent excitatory and inhibitory streams of input alone (without network effects or coincidence detectors) component inhibitory and excitatory currents must be on the order of 50 times larger than the net 
current. Physiological experiments rule out the existence of such extremely large hyperpolarizing or shunting currents in cortical cells (e.g., Nelson 1991; Douglas et al. 1988; Berman et al. 1991). A very fast, sodiumbased $^{5}$ spiking mechanism working at the millisecond scale and located in the dendritic tree can, in principle, enable cortical cells to respond as a coincidence detector resulting in high variability (Softky and Koch 1992, 1993). As of yet, no solid experimental evidence exists for such fast, powerful, all-or-none dendritic phenomena in cortical cells, although, given the relative inaccessibility of distal dendrites, they cannot be ruled out.

5.4 Correlation Functions. It is remarkable that the cross-correlation functions show a dual process: a sharp peak at small delay intervals followed by a much slower decay, characterized by a power law with exponent -0.21 to an asymptotic level. Exactly this form of cross-correlation was found to be the most common correlation structure in physiological recordings from cat visual cortex by Nelson and his colleagues (Nelson et al. 1992), who termed it a "castle on a hill" structure. Similar crosscorrelograms were reported among cells in the macaque inferotemporal cortex by (Gochin et al. 1991).

Our cross-correlograms are more peaked for units that are near each other and receive similar input from the surround; as the distance between the units increases, the central peak becomes wider and smaller, as reported in physiological studies (Nelson et al. 1992). Following Toyama et al. (1981), we computed the correlation coefficient defined as the fraction of spikes from two separate trains that are within $\pm 9 \mathrm{msec}$ of each other (the width of the central peak in the cross-correlogram Fig. 12b is $9 \mathrm{msec}$ ). This coefficient, measuring the degree to which two spike trains entrain each other, is 0.59 for adjacent cells and 0.29 for cells four lattice sites apart. When the distance between units corresponds to the characteristic inhibition range (here $d=9$; see Fig. 2), the cross-correlation shows a slight dip at short time scales.

It should be noted that the cross-correlation functions are peaked at zero time delay, in agreement with experimental results (Nelson et al. 1992). However, as opposed to classical "common input" interpretations in which neurons are hypothesized to share a common external input, the zero-delay peaks here are generated by recurrent excitation, leading to local clusters of excitation. The form of the cross-correlation functions (the "castle on a hill" structure) suggests that two processes are taking place; on a fast time scale (of less than $10 \mathrm{msec}$ ), the presence of excitatory clusters leads to synchronization, while on a slower time scale (up to $300 \mathrm{msec}$ ) the correlations are due to the clusters' diffusive trajectory.

\footnotetext{
${ }^{5}$ Calcium dendritic spikes are too slow to affect the neuronal variability at the time scale observed.
} 
5.5 Local Field Potentials. As illustrated in Figure 9, the excitatory current from the lateral connections impinging upon a cell shows strong fluctuations on a scale that is much faster than the characteristic time scale for the diffusion of clusters. These fluctuations are due to the internal dynamics of the activity inside a cluster (which oscillate, nonperiodically, in size). We measured a related signal, the total number of active cells within a circular region of radius 5 , and computed its power spectrum (Fig. 10). This can be thought of as the local field potential; it has a small peak around $40 \mathrm{~Hz}$. The power spectrum associated with the total spiking activity within a neighborhood of 9 units radius shows a much enhanced peak at $43 \mathrm{~Hz}$ (Fig. 11), corresponding to the $\gamma$ domain of slow wave activity in electrophysiology. Interestingly, the power spectra of spike trains of individual cells within the network (Fig. 7), as well as those of disconnected units (Fig. 7), show little evidence of a peak in this frequency band.

Furthermore, the "single unit" power spectrum (Fig. 7) shows a prominent $1 / f$ decay, while such a component-caused by the long-range fluctuations due to the network connectivity-is totally absent in the spectrum computed for the simulation of disconnected units (Fig. 7). Notice that in order to see such a decay for very low frequencies in the spectrum, single units must be recorded for on the order of $10-100 \mathrm{sec}$. This explains why they are not evident in the power spectra computed from $2 \mathrm{sec}$ long spike trains (Bair et al. 1994).

Not only does the $1 / f$ decay disappear as one moves from the power spectra of single units to the spectra of local field potentials (Figs. 10 and 11 ), but a peak in the $\gamma$ domain asserts itself. This change in the shape of the power spectra can be understood in terms of the autocorrelation function associated with the activity of the clusters.

Since the cluster activity is the sum of all single-unit spiking activity within a cluster of $N$ cells, the autocorrelation of the cluster spiking activity will be the sum of $N$ autocorrelations functions of the individual cells and $N \times(N-1)$ cross-correlation functions among individual cells within the cluster $(N \approx 261$ for our standard model). Thus, the autocorrelation of the entire cluster activity is dominated by the cross-correlations among individual units. It can be shown with the help of the Wiener-Khinchin theorem and the additivity of the Fourier transform that the power spectrum of the cluster activity will be dominated by the sum of the Fourier transforms of the cross-correlations between cells within the cluster (see Fig. 12). For pairs of cells at distances of 4 or less (such that they excite each other), the Fourier transforms of the cross-correlation functions (the 2 and 3 plots in Fig. 12) have a $1 / f$ peak at the origin, a small, wide peak at about $40 \mathrm{~Hz}$ (due to the secondary peak in the excitatory crosscorrelations), and a decline to zero for frequencies larger than $100 \mathrm{~Hz}$ (the reciprocal of the width of the central peak of the cross-correlations).

The amplitude of the Fourier transform of the correlation function of pairs of cells at $d=9$, that is, units that directly inhibit each other, 
shows an inverted $1 / f$ (negative) component, which decays asymptotically to zero. Thus, when the area over which the activity is averaged is sufficiently large to include such "inhibitory" interactions, here a circle of minimal radius equal to nine, many such inhibitory cell pairs are included. As a result the $1 / f$ component in the power spectrum of the radius 5 activity is canceled.

Two factors lead to a power spectrum peak in the $30-70 \mathrm{~Hz}$ range (Fig. 11). The first is the existence of secondary peaks around $25 \mathrm{msec}$ in the excitatory cross-correlations. The second is the relative width of the excitatory cross-correlation peaks to the inhibitory troughs. In general, the excitatory "castles" are sharp relative to the broad dip in the crosscorrelation due to inhibition. In Fourier space, these relationships are reversed: broader Fourier transforms of excitatory cross-correlations are paired with narrower Fourier transforms of inhibitory cross-correlations. Superposition of such transforms leads to a peak in the $30-70 \mathrm{~Hz}$ range.

Note that the peak around $40 \mathrm{~Hz}$ in the global activity develops in the absence of any explicit oscillators. The period of the "oscillations" we observe reflects the delay between the buildup of excitation in a cluster and the inhibitory response. This agrees with previous simulations of oscillations in cortex (Wilson and Bower 1991; Bush and Douglas 1991) and analytical results (Wilson and Cowan 1972; Koch and Schuster 1992) outlining the crucial role of inhibitory interactions in generating neuronal oscillations at the population level.

While cortical oscillations in the $30-$ to $90-\mathrm{Hz}$ range are commonly found in local field potential or multi-unit activity measurements in both cat and monkey visual cortex (Gray et al. 1990; Kreiter and Singer 1992), these oscillations are much less evident in single-unit data (Eckhorn et al. 1993; Eeckman and Freeman 1990; Young et al. 1992; Bair et al. 1994). We here offer a general explanation for this phenomenon.

5.6 Long-Range Fluctuations. We find that the firing of integrateand-fire units, embedded in a network with specific connectivity rules, shows clustering, long-term fluctuations, and self-similarity (fractal behavior) over time scales from 20 to $800 \mathrm{msec}$ (as based on the behavior of the variance versus mean curve in the number of events).

This fractal behavior is not a trivial result in our model but depends critically on the ratio of the weight of the inhibitory synaptic input to its excitatory counterpart. Thus, for $\beta=0.67$, the exponent $\nu$ in the law relating the mean number of spikes to the variability in this mean rate (equation 2.6) $\nu=1.4$, while for $\beta=0.50, \nu=1.0$, that is, identical to that expected for a Poisson process.

Moreover, our model accounts for the power law increase (with exponents $\nu$ between 1 and 2) for two different, and previously unrelated, experimental paradigms: repetitive short trials with varying sensory stimuli (Fig. 14) and trials with a stationary input and very long spike trains (Fig. 13). The similarity of the results (both showing power law rela- 
tions) suggests that these two types of fluctuations may have a common origin. For the model, one can assume that fluctuations in space across the network are equivalent to temporal fluctuations, that is, that a spatial average of single cell properties is equivalent to a temporal average over a long spike train from one cell. This assumption is known as ergodicity.

However, as this procedure involves a variation in the stimulus strength (affecting the mean rate $\lambda$ ) in a fixed time interval, it indicates a power law dependency of the correlation function, $A(\lambda, t)$, on $\lambda$ (equation 2.14), suggesting that the assumption that the normalized correlation is a function of the number of events, $\lambda t$, is a reasonable approximation.

The best power law fit $t^{-0.2}$ to the autocorrelation function predicts that the power spectrum will decay with a power law of $1 / f^{0.8}$, via the Wiener-Khinchin theorem. Indeed, the power spectrum (see Fig. 7) does behave as $1 / f^{0.8}$ at low frequencies.

Two implications of these results are worth noticing: First, these correlations decay slowly in time due to the small exponent of the power, leading to long temporal fluctuations. Practically, this means that these point processes do not behave as a Poisson process and averaging over spike trains of duration $T$ will not increase the signal-to-noise by $\sqrt{T}$. In the extreme case of $\nu=2$, the signal-to-noise is independent of the duration of the signal. For the nervous system this would imply that there is no penalty in performing computations based on the first few tens of milliseconds of spikes, rather than averaging over much longer times. Tovee et al. (1993) find that $50 \mathrm{msec}$ intervals from spike trains in visual cortex are sufficient to recover most of the information in the spike code, and that longer intervals carry little additional information.

Second, the theory of renewal processes (Lowen and Teich 1993) outlined in Section 2 predicts an exponent of -0.3 for the autocorrelation, which was confirmed by numerical simulations using the interval distribution of Figure 8 as the source for a renewal process. Higher order correlations between spikes lead to a slightly slower decay of the true autocorrelation than expected for a renewal process. In fact, Lowen and Teich (1992) suggest that such higher order correlations between interspike intervals are sufficient to produce $1 / f$-type power spectra in recordings from the auditory nerve; these spike trains, however, are no longer fractal point processes.

While the temporal range of correlations is limited by an upper cutoff of $300 \mathrm{msec}$, it is still remarkable that such a long time scale emerges from a system whose longest intrinsic time constant (the membrane time constant) is 20 msec. Changing model parameters can lead to an extended temporal range of correlation effects accompanied by lower firing rates. The underlying ISI distribution that gives rise to the autocorrelation is clearly power law (see Fig. 8), even though no clear distinction between power law and exponential decay can be made for the autocorrelation itself, since the range is limited. The exact nature of correlations in the discharge patterns of various cortical cells in behaving monkey should 
be the subject of future experimental investigation to test to what extent and over which time scale they show self-similar behavior.

The existence of $1 / f$ noise is generally considered a puzzle in most physical systems, which tend to exhibit temporal correlations on some characteristic time scale, leading to faster decay in the power spectrum. Recently a scenario leading to generic formation of $1 / f$ noise was proposed via systems that exhibit self-organized criticality, such as sand-piles and earthquakes (Bak et al. 1987; Olami et al. 1992). Poised at the brink of a dynamic phase transition point, self-organized critical systems can display many long-range behaviors. From the computational point of view, such systems have the advantage of very sensitive responses to small fluctuations. Our model is exquisitely sensitive to small local variations in the external input rate-a feature that is general to all models of selforganized criticality. The brain may employ such dynamic behavior to adapt to low signal-to-noise ratios of the afferent input to cortex. Whether the brain exhibits such self-organized criticality is an open question that should be explored in future research.

\section{Appendix A: Fractal Dimension of a Point Process}

The fractal dimension of a point process (dust) (Mandelbrot 1983) is defined by the number $n(\delta)$ of covering intervals of length $\delta$ needed to cover all the events occurring during a process of length $T$ :

$$
n(\delta) \propto \delta^{-D}
$$

The covering set $n(\delta)$, is obtained by the following procedure:

1. Divide a long process of length $L$ in $N(\delta)=L / \delta$ equal time intervals of length $\delta$.

2. Define $n(\delta)$ as the subset of intervals $N(\delta)$ that contains at least one event [i.e., the process is totally covered by $n(\delta)$ intervals of length $\delta]$.

3. The number of coverage intervals $n(\delta)$ is

$$
n(\delta)=\frac{N}{\delta}\left[1-P_{\text {empty }}(\delta)\right]
$$

where $P_{\text {empty }}(\delta)$ is the probability that a randomly chosen covering interval is empty.

4. $P_{\text {empty }}(\delta)$ can be calculated from the forward recurrence time distribution, $F_{w}(x)$. (For a randomly chosen sampling point, the forward recurrence time $w$ is the time duration between the sampling point 
and the first following event; $F_{w}(x)$ is the probability distribution of $w$.)

$$
P_{\text {empty }}(\delta)=\int_{\delta}^{\infty} F_{w}(x) d x
$$

(every coverage interval whose left edge has a forward recurrence time $w>\delta$ is necessarily empty).

5. For a general stationary point process, $F_{w}(x)$ is (Cox and Lewis 1966)

$$
F_{w}(x)=A R(x)=A \int_{x}^{\infty} P(x) d x
$$

where $P(x)$ is the interval distribution probability, $A$ is a normalization factor for $F_{w}$, and $R(x)$, the integral of $\mathrm{P}(\mathrm{x})$, is the survivor probability for $x[\operatorname{Prob}(T>x)]$.

In the following we calculate $D$ for a point process characterized by an interval probability distribution, $P(t)$, which decays as a power law with exponent $1<\gamma<2$ :

$$
P(t)=B t^{-\gamma} \quad t>\Lambda
$$

for $\Lambda<t<T$ and zero otherwise (the cut-off $T \rightarrow \infty$ is assumed for regularization).

The corresponding survivor function is:

$$
R(x)= \begin{cases}1 & \text { for } x<\Lambda \\ \frac{B x^{-\gamma+1}}{\gamma-1} & \text { for } \Lambda<x<T\end{cases}
$$

The normalized $F_{w}$ (equation 2.5 ) is

$$
F_{w}(x)=\frac{-\delta+2}{T^{-\delta+2}} x^{-\gamma+1} \Lambda<x<T
$$

From this, $P_{\text {empty }}$ can be obtained (equation A.3):

$$
P_{\text {empty }}(\delta)=1-\frac{\delta}{T}^{-\gamma+2}
$$

The number of coverage intervals $n(\delta)$ is (equation A.2)

$$
n(\delta)=\frac{L}{\delta} \frac{\delta}{T}^{-\gamma+2} \propto \delta^{-\gamma+1}
$$

In conjunction with equation A.1, this implies that the fractal dimension is $D=\gamma-1$. Following the same steps, the dimension of a Poisson process (exponential interval distribution) can be shown to be $D=1$. 


\section{Appendix B: Relating the Variance to the Autocorrelation}

The proof of equation 2.8 follows Cox and Lewis (1966).

To determine the variance in the number of events in time $T$, partition the window $T$ into $n$ bins (of width one millisecond). Let $X_{i}$ be the random variable describing the number of events in bin number $i$. Then the variance is

$$
\begin{aligned}
\operatorname{Var}(T) & =\operatorname{Var}\left(X_{1}+X_{2}+\cdots+X_{n}\right) \\
& =E\left[\left(X_{1}+X_{2}+\cdots+X_{n}\right)^{2}\right]-\left[E\left(X_{1}+X_{2}+\cdots+X_{n}\right)\right]^{2} \\
& =2 \sum_{l=1}^{n-1} \sum_{k>1}^{n}\left[E\left(X_{k} X_{l}\right)-E\left(X_{k}\right) E\left(X_{l}\right)\right]+\sum_{i=1}^{n}\left[E\left(X_{i}^{2}\right)-E\left(X_{i}\right)^{2}\right] \\
& =2 \sum_{l=1}^{n-1} \sum_{k=l+1}^{n} \operatorname{Cov}\left(X_{l}, X_{k}\right)+n \operatorname{Var}\left(X_{i}\right)
\end{aligned}
$$

If the bin size is so small that the possibility of multiple spikes in the same bin is excluded, then the variance in the number of spikes in that interval is equal to the mean, $\operatorname{Var}\left(X_{i}\right)=E\left(X_{i}\right)$. Replacing $\operatorname{Var}\left(X_{i}\right)$ and changing the indices on the sum,

$$
\begin{aligned}
\operatorname{Var}(T) & =2 \sum_{l=1}^{n-1} \sum_{k=1}^{n-l} \operatorname{Cov}\left(X_{i}, X_{i+k}\right)+n E\left(X_{i}\right) \\
& =2 \sum_{l=1}^{n-1}(n-l) \operatorname{Cov}\left(X_{i}, X_{i+l}\right)+n E\left(X_{i}\right)
\end{aligned}
$$

Since we are interested in the limit for which $T \gg 1 \mathrm{msec}$, the covariance will tend toward the autocorrelation function minus the chance level of correlation,

$$
\operatorname{Cov}\left(X_{i}, X_{i+l}\right) \rightarrow A\left(\tau_{l}\right)-A(\infty)
$$

In this limit, one can replace the sum with an integral over the correlation. With $n E\left(X_{i}\right)=N(T)$, we obtain

$$
\operatorname{Var}(T)=N(T)\left\{1+\frac{2}{\lambda T} \int_{0}^{T}(T-\tau)[A(\tau)-A(\infty)] d \tau\right\}
$$

which is equation 2.8 .

\section{Acknowledgments}

We thank William Softky for many invaluable comments and ideas regarding the origin and the function of the high variability in cortical cells. We are also indebted to Terry Sejnowski, Wyeth Bair, and Ernst Niebur for insightful discussions. Our research was supported by a $\mathrm{My}$ ron A. Bantrell Research Fellowship, the Howard Hughes Medical Institute, the National Science Foundation, the Office of Naval Research, and the Air Force Office of Scientific Research. 


\section{References}

Abeles, M. 1982. Role of the cortical neuron: integrator or coincidence detector? Israel J. Med. Sci. 18, 83-92.

Abeles, M. 1991. Corticonics-Neural Circuits of the Cerebral Cortex. Cambridge University Press, Cambridge.

Amari, S.-I. 1977. Dynamics of pattern formation in lateral-inhibition type neural fields. Biol. Cybern. 27, 77-87.

Amit, D. J., and Tsodyks, M. V. 1991a. Quantitative study of attractor neural network retrieving at low rates: 1 . Substrate spikes, rates and neuronal gain. Network Com. 2(3), 259-273.

Amit, D. J., and Tsodyks, M. V. 1991b. Quantitative study of attractor neural network retrieving at low rates: 2 . Low-rate retrieval in symmetrical networks. Network Com. 2(3), 275-294.

Asanuma, H., and Rosen, I. 1973. Spread of mono- and polysynaptic connections within cat's motor cortex. Exp. Brain Res. 16, 507-520.

Bair, W., Koch, C., Newsome, W., and Britten, K. 1994. Power spectrum analysis of MT neurons in the behaving monkey. J. Neurosci., in press.

Bak, P., Tang, C., and Wiesenfeld, K. 1987. Self-organized criticality: An explanation of $1 / \mathrm{f}$ noise. Phys. Rev. Lett. 59, 381-384.

Berman, N. J., Douglas, R. J., Martin, K. A. C., and Whitteridge, D. 1991. Mechanisms of inhibition in cat visual cortex. J. Physiol. 440, 697-722.

Bernander, Ö., Koch, C., and Usher, M. 1994. The effect of synchronized inputs at the single neuron level. Neural Comp. 6, 622-641.

Bush, P. C., and Douglas, R. J. 1991. Synchronization of bursting action potential discharge in a model network of neocortical neurons. Neural Comp. 3, 19-30.

Chernjavsky, A., and Moody, J. 1990. Spontaneous development of modularity in simple cortical models. Neural Comp. 2(3), 334-354.

Cowan, J. D. 1982. Spontaneous symmetry breaking in large scale nervous activity. Int. J. Quant. Chem. 22, 1059-1082.

Cox, D., and Lewis, P. A. W. 1966. The Statistical Analysis of Series of Events. Chapman and Hall, London.

Douglas, R. J., and Martin, K. A. C. 1990. Neocortex. In The Synaptic Organization of the Brain, G. M. Shepherd, ed., pp. 389-438. Oxford Univ. Press, New York.

Douglas, R. J., Martin, K. A. C., and Whitteridge, D. 1988. Selective responses of visual cortical-cells do not depend on shunting inhibition. Nature (London) 332(6165), 642-644.

Eckhorn, R., Frien, A., Bauer, R., Woelbern, T., and Harald, K. 1993. High frequency $(60-90 \mathrm{hz})$ oscillations in primary visual cortex of awake monkey. Neuroreport 4, 243-246.

Eeckman, F., and Freeman, W. 1990. Correlations between unit firing and EEG in the rat olfactory system. Brain Res. 528(2), 238-244.

Ermentrout, G. B., and Cowan, J. D. 1980. Large scale spatially organized activity in neural nets. SIAM J. Appl. Math. 38, 1-21.

Feder, J. 1988. Fractals. Plenum Press, New York.

Fetz, E., Toyama, K., and Smith, W. 1991. Synaptic interactions between cortical 
neurons. In Cerebral Cortex, Vol. 9, A. Peters and E. G. Jones, eds., pp. 1-48. Plenum Press, New York.

Gochin, P., Miller, E., Gross, C., and Gerstein, G. 1991. Functional interactions among neurons in inferior temporal cortex of the awake macaque. Exp. Brain Res. 84, 505-516.

Gray, C. M., Engel, A. K., König, P., and Singer, W. 1990. Stimulus dependent neuronal oscillations in cat visual cortex: Receptive field properties and feature dependence. Eur. J. Neurosci. 2, 607-619.

Grinvald, A. 1992. Optical imaging of architecture and function in the living brain sheds new light on cortical mechanisms underlying visual perception. Brain Topogr. 5(2), 71-75.

Grueneis, F., Nakao, M., and Yamamoto, M. 1990. Counting statistics of $1 / \mathrm{f}$ fluctuations in neuronal spike trains. Biol. Cybern. 62, 407-413.

Hess, R., Negishi, K., and Creutzfeldt, O. 1975. The horizontal spread of intracortical inhibition in the visual cortex. Exp. Brain Res. 22, 415-419.

Knight, B. 1972. Dynamics of encoding in a population of neurons. J. Gen. Physiol. 59, 734-766.

Koch, C., and Schuster, H. 1992. A simple network showing burst synchronization without frequency-locking. Neural Comp. 4, 211-223.

Komatsu, Y., Nakajima, S., Toyama, K., and Fetz, E. E. 1988. Intracortical connectivity revealed by spike-triggered averaging in slice preparations of cat visual cortex. Brain Res. 442(2), 359-362.

Kreiter, A. K., and Singer, W. 1992. Oscillatory neuronal responses in the visual cortex of the awake macaque monkey. Eur. J. Neurosci. 4, 369-375.

Lowen, S. B., and Teich, M. C. 1992. Auditory-nerve action potentials form a nonrenewal point process at short as well as long time scales. J. Acoust. Soc. Am. 92(2), 803-806.

Lowen, S. B., and Teich, M. C. 1993. Fractal renewal processes generate 1/f noise. Phys. Rev. E 47(2), 992-1001.

Mandelbrot, B. B. 1983. The Fractal Geometry of Nature. W. H. Freeman, New York.

Mason, A., Nicoll, A., and Stratford, K. 1991. Synaptic transmission between individual neurons of the rat visual cortex in vitro. J. Neurosci. 11, 72-84.

Nelson, J. I., Salin, P. A., Munk, M. H.-J., Arzi, M., and Bullier, J. 1992. Spatial and temporal coherence in cortico-cortical connections: A cross-correlation study in areas 17 and 18 in the cat. Visual Neurosci. 9, 21-38.

Nelson, S. B. 1991. Temporal interactions in the cat visual system. iii. Pharmacological studies of cortical suppression suggest a presynaptic mechanism. J. Neurosci. 1991(11), 369-380.

Olami, Z., Feder, H. J. S., and Christensen, K. 1992. Self-organized criticality in a continuous, nonconservative cellular automaton modeling earthquakes. Phys. Rev. Lett. 68(8), 1244-1247.

Orbach, H. S., Felleman, D. J., Ribak, E. N., and Van Essen, D. C. 1992. Visualization of cortical connections with voltage sensitive dyes. In Analysis and Modeling of Neural Systems, F. H. Eeckman, ed., pp. 15-28. Kluwer Academic, Norwell, MA.

Shofner, W. P., and Dye, R. H. J. 1989. Statistical and receiver operating char- 
acteristic analysis of empirical spike-count distributions: Quantifying the ability of cochlear nucleus units to signal intensity changes. J. Acoust. Soc. Am. 86, 2172-2184.

Silva, L. R., and Connors, B. W. 1987. Spatial distribution of intrinsic cortical neurons that excite or inhibit layer II/III cells: A physiological study of the neocortex in vitro. Soc. Neurosci. Abst. 12, 1435.

Snowden, R. J., Treue, S., and Andersen, R. A. 1992. The response of neurons in areas V1 and MT of alert rhesus monkey to moving dot patterns. Exp. Brain Res. 88, 389-400.

Softky, W. 1993. Sub-millisecond coincidence detection in active dendritic trees. Neuroscience 58(1), 13-41.

Softky, W. R., and Koch, C. 1992. Cortical cells should fire regularly, but do not. Neural Comp. 4(5), 643-645.

Softky, W. R., and Koch, C. 1993. The highly irregular firing of cortical cells is inconsistent with temporal integration of random EPSPs. J. Neurosci. 13(1), 334-350.

Stevens, C. F. 1993. What form should a cortical theory take. In Large-Scale Neuronal Theories of the Brain, C. Koch and J. Davis, eds. MIT Press, Boston.

Teich, M. C. 1989. Fractal character of the auditory neural spike train. IEEE Trans. Biomed. Eng. 36(1), 150-160.

Teich, M. C. 1992. Fractal neuronal firing patterns. In Single Neuron Computation, T. McKenna, J. Davis, and S. F. Zornetzer, eds., pp. 589-625. Academic Press, San Diego, CA.

Tolhurst, D., Movshon, J., and Dean, A. 1983. The statistical reliability of signals in single neurons in cat and monkey visual cortex. Vision Res. 23, 775-785.

Tovee, M. J., Rolls, E. T., and Treves, A. 1993. Information encoding and the responses of single neurons in the primate temporal visual-cortex. J. Neurophysiol. 70(2), 640-654.

Toyama, K., Kimura, M., and Tanaka, K. 1981. Organization of cat visual cortex as investigated by cross-correlation technique. J. Neurophysiol. 46(2), 202214.

Tsodyks, M., Mitkov, I., and Sompolinsky, H. 1993. Pattern of synchrony in inhomogeneous networks of oscillaters with pulse interactions. Phys. Rev. Lett. 71(8), 1280-1283.

Tuckwell, H. C. 1988. Introduction to Theoretical Neurobiology. Cambridge University Press, New York.

van Vreeswijk, C., and Abbott, L. F. 1993. Self-sustained firing in populations of integrate-and-fire neurons. SIAM J. Appl. Math. 53(1), 253-264.

Vogels, R., Spileers, W., and Orban, G. A. 1989. The response variability of striate cortical neurons in the behaving monkey. Exp. Brain Res. 77, 432-436.

Werner, G., and Mountcastle, V. B. 1963. The variability of central neural activity in a sensory system and its implications for the central reflection of sensory events. J. Neurophysiol. 26, 958-977.

Willshaw, D., and von der Malsburg, C. 1976. How patterned neural connections can be set up by self-organization. Proc. R. Soc. London B 194, 431-445.

Wilson, H. R., and Cowan, J. D. 1972. Excitatory and inhibitory interactions in localized populations of model neurons. Biophys. J. 12, 1-24. 
Wilson, H. R., and Cowan, J. D. 1973. A mathematical theory of the functional dynamics of cortical and thalamic nervous tissue. Biol. Cybern. 13, 55-80.

Wilson, M. A., and Bower, J. M. 1991. A computer simulation of oscillatory behavior in primary visual cortex. Neural Comp. 3, 498-509.

Yamada, W. M., Koch, C., and Adams, P. R. 1989. Multiple channels and calcium dynamics. In Methods in Neuronal Modeling, C. Koch and I. Segev, eds., pp. 97-133. MIT Press, Cambridge, MA.

Young, M., Tanaka, K., and Yamane, S. 1992. Oscillatory neuronal responses in the visual cortex of the monkey. J. Neurophysiol. 67, 1464-1474.

Zhou, Y., and Fuster, J. 1992. Unit discharge in monkey's parietal cortex during perception and mnemonic retention of tactile features. Soc. Neurosci. Abstract 18(1), 706.

Zipser, D., Kehoe, B., Littlewort, G., and Fuster, J. 1993. A spiking network model of short-term active memory. J. Neurosci., in press.

Received August 24, 1993; accepted November 5, 1993. 